\title{
INDIFFERENCE TO SYMMETRY IN HRUSHOVSKI'S AB INITIO CONSTRUCTION
}

\author{
OMER MERMELSTEIN
}

\begin{abstract}
Denote Hrushovski's non-collapsed ab initio construction for an $n$-ary relation by $\mathcal{M}_{\nsim}$ and the analogous construction for a symmetric $n$-ary relation by $\mathcal{M}_{\sim}$. We show that $\mathcal{M}_{\nsim}$ is isomorphic to a proper reduct of $\mathcal{M}_{\sim}$ and vice versa, and that the combinatorial pregeometries associated with both structures are isomorphic.
\end{abstract}

\section{INTRODUCTION}

The source of pregeometries in model theory is the closure operation on realizations of a regular type, given by forking. The best known example of this, is the algebraic closure operation on a strongly minimal set. In the early 1980s Boris Zilber conjectured that, after naming some parameters, the geometry of a strongly minimal set is isomorphic to that of a set, that of a vector space or that of an algebraically closed field. This conjecture was refuted by a construction introduced by Ehud Hrushovski in [Hru93], referred to as Hrushovski's ab initio construction.

Hrushovski's ab initio construction is a deep generalization of a Fraïssé limit featuring a regular type whose forking geometry is non-disintegrated, yet prohibiting the existence of an infinite definable group. By imposing restrictions on the class from which the limit is constructed, one produces a strongly minimal structure not falling within Zilber's conjecture. Lifting these restrictions on the class produces an $\omega$-stable limit of Morley rank $\omega$, whose unique type of rank $\omega$ is regular and of the same geometrical flavor. The $\omega$-stable version of the construction is often referred to as the non-collapsed version in contrast to the collapsed strongly minimal version.

The innovative component of the construction, and the one that produces the desired geometry in the limit, is a combinatorial predimension function defined on the amalgamation class used, which determines the dimension function of the non-forking geometry of the limit. This idea has been used since then to construct many structures of a similar flavor. The purpose of this paper is to initiate a discussion regarding reduction relations (in the sense of reducts) between structures constructed using Hrushovski's techniques, and their respective geometries. In the algebraic "classic" strongly minimal structures, we know of strong ties between the structure's geometry and what the structure must interpret [Hru87, Rab93].

In addition to the ab initio constructions, using similar amalgamation methods, Hrushovski introduced [Hru92] a way to fuse together strongly minimal theories (with DMP) $T_{1}, T_{2}$ in disjoint languages $\mathcal{L}_{1}, \mathcal{L}_{2}$ into a strongly minimal $\mathcal{L}_{1} \cup \mathcal{L}_{2^{-}}$ theory $T^{*}$ whose every model has as reducts (in the obvious way) both a model of

2010 Mathematics Subject Classification. Primary 03C30; Secondary 03C45.

Key words and phrases. Hrushovski construction, predimension, reduct, pregeometry. 
$T_{1}$ and a model of $T_{2}$. In fact, if the languages $\mathcal{L}_{1}$ and $\mathcal{L}_{2}$ are known, $T^{*}$ can be characterized (up to a multiplicity function $\mu$ ) by these reducts. Our results were partially motivated by the resemblance of the ab initio construction to the fusion construction, and the hope to shed light on what other reducts could appear in $T^{*}$ due to the amalgamation process.

1.1. Structure and results. Prior to committing to any particular amalgamation classes, we describe general procedures for lifting maps between free amalgamation classes $\mathbb{C}_{1}, \mathbb{C}_{2}$ (arising from predimension functions) to relations between their generic structures $\mathcal{M}_{1}, \mathcal{M}_{2}$. In particular, Assumption 2.16 provides a sufficient condition for $\mathcal{M}_{2}$ to be isomorphic to a proper definable reduct of $\mathcal{M}_{1}$.

To state our main result, we recall that Hrushovski's ab initio construction from [Hru93] is in the language of a relation symbol $R$ and consisting of a countable structure such that any finite substructure $A$ has at most $|A|$ many distinct instances of $R$. Two parameters that may be easily varied in the construction are $n$, the arity of the relation $R$, and the amount of symmetry $R$ admits. For a subgroup $\mathfrak{g} \leq S_{n}$, we let $\mathcal{M}_{\mathfrak{g}}$ be the generic model of Hrushovski's non-collapsed $n$-ary construction (see Section 3) where a single "instance" of $R$ is a $\mathfrak{g}$-orbit of an $n$-tuple. For better readability, we denote the subgroup $S_{n}$ by $\sim$, and the subgroup $\{i d\}$ by $\nsim$. Hence, in $\mathcal{M}_{\nsim}$ the relation $R$ has no intrinsic symmetry, in $\mathcal{M}_{\sim}$ the relation $R$ is fully symmetric, and for an arbitrary subgroup $\mathfrak{g}, \mathcal{M}_{\mathfrak{g}}$ is "in between" the two in terms of symmetry. Theorem 3.15 shows in a direct manner that for $\mathfrak{h} \subseteq \mathfrak{g}$, the structure $\mathcal{M}_{\mathfrak{g}}$ can naturally be seen as a reduct of $\mathcal{M}_{\mathfrak{h}}$, i.e., a larger amount of symmetry corresponds to a "coarser" reduct. It is a-priori unclear whether one can go in the other direction - find a "coarser" reduct which is less symmetric.

Using the methods of Section 2, we obtain the main theorem, which essentially states that - from the point of view of reduction and pregeometry - varying symmetry is inconsequential. This is unlike the arity of the relation $R$, which does affect the pregeometry of the resulting structure [EF11].

Theorem 3.9. For all $\mathfrak{g}, \mathfrak{h} \leq S_{n}$, the pregeometries associated to $\mathcal{M}_{\mathfrak{g}}$ and $\mathcal{M}_{\mathfrak{h}}$ are isomorphic, and $\mathcal{M}_{\mathfrak{h}}$ is isomorphic to a proper definable reduct of $\mathcal{M}_{\mathfrak{g}}$.

The theorem, besides a proof-of-concept of the usefulness of the methods of Section 2 , is actually itself quite useful, from a technical point of view, for our future endeavors. When attempting to prove or deny the existence of a reduct with a certain property, one may choose the level of symmetry with which to work. For example in [Mer13], where a given generic structure $\mathcal{N}$ (distinct from the structures $\mathcal{M}_{\mathfrak{g}}$ discussed here) is shown to be isomorphic to the reduct of $\mathcal{M}_{\sim}$ to a certain formula $\varphi$, much of the effort revolves around making sure no "unintentional" realizations of $\varphi$ are introduced due to the symmetry of the structure. When looking at the reduct given by the same formula $\varphi$ in $\mathcal{M}_{\nsim}$, the complexity vanishes and mere straightforward investigation yields that the obtained reduct is indeed $\mathcal{N}$. Thus, it suffices to study $\mathcal{M}_{\nsim}$ in order to conclude that $\mathcal{N}$ is a reduct of $\mathcal{M}_{\sim}$. Similarly, the results of [HM19] (which also employs the methods developed here ${ }^{1}$ ), pertaining to reducts of $\mathcal{M}_{\nsim}$ with non-disintegrated pregeometries distinct from that of $\mathcal{M}_{\nsim}$, apply to any $\mathcal{M}_{\mathfrak{g}}$.

\footnotetext{
${ }^{1}$ Though technically published at a later date, the results of the current paper precede the inception of [HM19].
} 
Additionally, the discovery that $\mathcal{M}_{\nsim}$ is a proper reduct of $\mathcal{M}_{\sim}$, and not only the other way around, has an immediate consequence that, for arity strictly greater than 2, there is an infinite descending chain of proper reducts with non-disintegrated geometries, beginning with $\mathcal{M}_{\sim}$. From this, we get the (weaker) result that there is a strictly ascending chain of closed subgroups of $S_{\infty}$ beginning with the automorphism group of any $\mathcal{M}_{\mathfrak{g}}$. Although not answering any specific asked question, this result is in spirit with exploration of group-reducts of Hrushovski constructions. In an unpublished work titled "Some 'group reducts' of Hrushovski structures" Ghadernezhad explores the number of group-reducts of various Hrushovski constructions, and in [KS16] Kaplan and Simon ask whether the automorphism group of the geometry of a certain Hrushovski construction is maximal.

Although we believe this to be the case, we do not explore in this paper whether analogues of our result hold for the strongly minimal version of Hrushovski's ab initio construction. Doing so would introduce a significant amount of technical complication, which this paper already does not lack. This choice is further motivated by Evans and Ferreira's [EF12, Section 3] result that the pregeometry of the non-collapsed construction is (under minor - for our purposes - technical assumptions) isomorphic to that of the collapsed strongly minimal construction.

\section{Free amalgamation Classes given by a Predimension}

We use calligraphic capital letters for first-order structures and their roman counterpart for the universe of the structure, i.e., $M$ is the universe of the structure $\mathcal{M}$. We denote the substructure induced by $\mathcal{M}$ on a subset $X \subseteq M$ by $\mathcal{M}[X]$. For an $\mathcal{L}$-structure $\mathcal{M}$ and a symbol $S \in \mathcal{L}$, we denote by $S^{\mathcal{M}}$ the interpretation of $S$ in $\mathcal{M}$. For an $\mathcal{L}$-formula $\varphi(\bar{x})$ (possibly with parameters from $\mathcal{M}$ ), we denote the set of realizations in $\mathcal{M}$ by $\varphi(\mathcal{M}):=\{\bar{a} \in M: \mathcal{M} \models \varphi(\bar{a})\}$.

We write $X \subseteq$ fin $Y$ to mean $X$ is a finite subset (or substructure) of $Y$. When listing sets and/or elements consecutively, we mean this as shorthand for union, where elements are taken to be singletons. For example, $A B x:=A \cup B \cup\{x\}$.

Definition 2.1. In the context of some language $\mathcal{L}$, for a class of finite relational $\mathcal{L}$-structures $\mathbb{C}_{0}$ closed under isomorphism and substructures, say that $\delta: \mathbb{C}_{0} \rightarrow \mathbb{Z}$ is a predimension function for $\mathbb{C}_{0}$ if

(1) $\delta$ is preserved under isomorphism

(2) $\delta(\emptyset)=0$

(3) $\delta(\mathcal{A}) \leq|A|$

(4) $\delta$ is submodular.

That is, for $\mathcal{D} \in \mathbb{C}_{0}$, for every $X, Y \subseteq D$

$$
\delta(\mathcal{D}[X \cup Y]) \leq \delta(\mathcal{D}[X])+\delta(\mathcal{D}[Y])-\delta(\mathcal{D}[X \cap Y]) .
$$

Notation 2.2. For any class $\mathbb{C}_{0}$ and a predimension $\delta$ as in the above definition, $\mathcal{D} \in \mathbb{C}_{0}$, and $X, Y \subseteq D$, we use the following shorthand notation:

- $\delta_{\mathcal{D}}(X)=\delta(\mathcal{D}[X])$

- $\delta_{\mathcal{D}}(Y / X)=\delta_{\mathcal{D}}(X \cup Y)-\delta_{\mathcal{D}}(X)$.

Thus, the submodularity of $\delta$ can be restated as $\delta_{\mathcal{D}}(Y / X) \leq \delta_{\mathcal{D}}(Y / X \cap Y)$.

Additionally, with the addition of a bar, we denote the class of structures whose every finite countable substructure is in $\mathbb{C}_{0}$, i.e.,

- $\overline{\mathbb{C}}_{0}=\left\{\mathcal{M}:|M| \leq \aleph_{0},\left\{\mathcal{A}: \mathcal{A} \subseteq_{\text {fin }} \mathcal{M}\right\} \subseteq \mathbb{C}_{0}\right\}$. 
In particular, $\mathbb{C}_{0} \subseteq \overline{\mathbb{C}}_{0}$.

Remark 2.3. In this paper, every class $\mathbb{C}$ discussed will have a predimension function associated with it. Similarly, each structure $\mathcal{M}$ will be seen as being in the context of some such class, and therefore have a unique predimension function associated with it, as well as with all its substructures. Thus, it will always make sense to use $\delta_{\mathcal{M}}(X)$ for a structure $\mathcal{M}$ and a subset $X \subseteq_{\text {fin }} M$, or any notions derived from $\delta_{\mathcal{M}}$, such as those defined in definitions 2.4, 2.7 and 2.8.

A statement of the form $\mathcal{M} \in \mathbb{C}$ or $\mathcal{M} \in \overline{\mathbb{C}}$, for an established class $\mathbb{C}$, encompasses that the predimension associated to $\mathcal{M}$ is the predimension of $\mathbb{C}$, and so disambiguates the meaning of $\delta_{\mathcal{M}}$. Later, we will vary languages so each of the discussed classes is in a distinct language, preventing confusion in contextualizing a structure.

Definitions $2.4-2.10$ below depend on a choice of $\mathbb{C}_{0}$, a class of finite structures closed under isomorphism and substructures, and $\delta$, a predimension function for $\mathbb{C}_{0}$. For the moment, fix such $\mathbb{C}_{0}$ and $\delta$.

Definition 2.4. For $\mathcal{M} \in \overline{\mathbb{C}}_{0}$ and $\mathcal{N} \subseteq \mathcal{M}$, say that $\mathcal{N}$ is self-sufficient (or strong) in $\mathcal{M}$ if for every $X \subseteq_{\text {fin }} M, \delta_{\mathcal{M}}(X / X \cap N) \geq 0$. Denote this by $\mathcal{N} \leqslant \mathcal{M}$. Write $N \leqslant \mathcal{M}$ to mean $N \subseteq M$ and $\mathcal{M}[N] \leqslant \mathcal{M}$.

A strong embedding is an embedding $f: \mathcal{N} \rightarrow \mathcal{M}$ such that $f[N] \leqslant \mathcal{M}$.

Remark 2.5. We linger on the notation $N \leqslant \mathcal{M}$ (and $\mathcal{N} \leqslant \mathcal{M}$ ) introduced in the definition above. There, $N$ is a capital letter, representing a set, and $\mathcal{M}$ is calligraphic letter, hence, representing a structure. This is in contrast with $\leqslant$ being defined above as a relation between two structures, and the meaning of $\leqslant$ requiring knowledge of some ambient $\delta$.

The way the statement $N \leqslant \mathcal{M}$ should be read is as information about a single structure $\mathcal{M}$, pertaining to how one of its subsets is situated. Recalling Remark 2.3, $\mathcal{M}$ carries a predimension $\delta_{\mathcal{M}}$ defined on every finite subset of its universe. With $\delta_{\mathcal{M}}$ well defined, there is no ambiguity in the definition of self-sufficiency within $\mathcal{M}$. Furthermore, there is no ambiguity regarding the structure on $N$, as we implicitly imbue the set $N$ with the structure and predimension induced on it by $\mathcal{M}$.

Formally, as we will often work in the context of two distinct notions of selfsufficiency, the specific interpretation of the $\leqslant$ relation alluded to in the statement $N \leqslant \mathcal{M}$ (or $\mathcal{N} \leqslant \mathcal{M}$ ) is inferred from $\delta_{\mathcal{M}}$. As further clarification, we will use different notation for self-sufficiency associated to different classes, e.g., $\leqslant *$ for $\mathbb{C}_{*}$.

As a rule, we are interested not in the full class $\mathbb{C}_{0}$, but only those structures $\mathcal{A} \in \mathbb{C}_{0}$ for which $\delta_{\mathcal{A}}$ is hereditarily non-negative. Notationally, we mark this restriction by removing the 0 subscript.

Definition 2.6. Define $\mathbb{C}=\left\{\mathcal{A} \in \mathbb{C}_{0}: \emptyset \leqslant \mathcal{A}\right\}$ and $\overline{\mathbb{C}}=\left\{\mathcal{M} \in \overline{\mathbb{C}}_{0}: \emptyset \leqslant \mathcal{M}\right\}$.

A consequence of the submodularity of $\delta$ is that the relation $\leqslant$ is transitive, and the intersection of self-sufficient subsets is self-sufficient. This implies that every subset $X$ of a structure $\mathcal{M} \in \overline{\mathbb{C}}_{0}$ has a unique self-sufficient closure in $\mathcal{M}$ the intersection of all self-sufficient subsets of $\mathcal{M}$ containing $X$. If $\mathcal{M} \in \overline{\mathbb{C}}$ (i.e., $\emptyset \leqslant \mathcal{M})$ and $X$ is finite, then the self-sufficient closure of $X$ is also finite.

Definition 2.7. For $\mathcal{M} \in \overline{\mathbb{C}}_{0}$ and $X \subseteq M$, define $\Lambda_{\mathcal{M}}(X)=\bigcap\{N \leqslant \mathcal{M}: N \supseteq X\}$, the self-sufficient closure of $X$ in $\mathcal{M}$. 
Prior to defining the dimension function associated to a structure $\mathcal{M}$, we recall that a combinatorial pregeometry (also known as a finitary matroid) is a set $M$ accompanied by a closure operator $\mathrm{cl}: \mathrm{P}(M) \rightarrow \mathrm{P}(M)$ with the exchange property (i.e. $x \in \operatorname{cl}(A y) \backslash \operatorname{cl}(A) \Longrightarrow y \in \operatorname{cl}(A x)$ ) where for every infinite $X \subseteq M$, the closure of $X$ is $\bigcup\left\{\operatorname{cl}\left(X_{0}\right): X_{0} \subseteq\right.$ fin $\left.X\right\}$. A set $X \subseteq M$ is closed in the pregeometry if $\operatorname{cl}(X)=X$. Every pregeometry has an associated dimension function d defined by $\mathrm{d}(Y)=\min \{|X|: Y \subseteq \operatorname{cl}(X)\}$. The closure operator can be defined from d by taking $\operatorname{cl}(X)=\{a \in M: \mathrm{d}(X a)=\mathrm{d}(X)\}$ whenever $X$ is finite. An isomorphism of pregeometries is a dimension-preserving (or, equivalently, closure-preserving) bijection between two pregeometries.

Definition 2.8. For a structure $\mathcal{M} \in \overline{\mathbb{C}}$, for each $X \subseteq_{\text {fin }} M$ define $\mathrm{d}_{\mathcal{M}}(X)=$ $\min \left\{\delta_{\mathcal{M}}(Y): X \subseteq Y \subseteq_{\text {fin }} M\right\}$ or, equivalently, $\mathrm{d}_{\mathcal{M}}(X)=\delta\left(\Lambda_{\mathcal{M}}(X)\right)$. For a countably infinite $N \subseteq M$ define $\mathrm{d}_{\mathcal{M}}(N)=\sup \left\{\mathrm{d}_{\mathcal{M}}(X): X \subseteq_{\text {fin }} N\right\}$.

The function $\mathrm{d}_{\mathcal{M}}$ in the above definition is the dimension function of a pregeometry $\left(M, \mathrm{cl}_{\mathcal{M}}\right)$, which we denote $\operatorname{PG}(\mathcal{M})$. We say that $\operatorname{PG}(\mathcal{M})$ is the pregeometry naturally associated to $\mathcal{M}$ via $\delta$.

Observation 2.9. The $\leqslant$ relation can be characterized in terms of associated pregeometries - whenever $\mathcal{N} \subseteq \mathcal{M} \in \overline{\mathbb{C}}$, the substructure $\mathcal{N}$ is self-sufficient in $\mathcal{M}$ if and only if $\mathrm{d}_{\mathcal{N}}$ is a restriction of $\mathrm{d}_{\mathcal{M}}$, i.e., $\operatorname{PG}(\mathcal{N}) \subseteq \operatorname{PG}(\mathcal{M})$.

Definition 2.10. Given structures $\mathcal{A}, \mathcal{B}_{1}, \mathcal{B}_{2} \in \overline{\mathbb{C}}_{0}$ such that $\mathcal{A} \leqslant \mathcal{B}_{1}$ and $\mathcal{A} \leqslant \mathcal{B}_{2}$, say that $\mathcal{D}$ is an amalgam of $\mathcal{B}_{1}$ and $\mathcal{B}_{2}$ over $\mathcal{A}$ if there exist strong embeddings $f_{i}: \mathcal{B}_{i} \rightarrow \mathcal{D}$ such that $f_{1}\left|A=f_{2}\right| A$.

If, in addition, $f_{1}\left[B_{1}\right] \cap f_{2}\left[B_{2}\right]=f_{1}[A]$, and the equality

$$
\delta_{\mathcal{D}}\left(X / X \cap f_{1}[A]\right)=\delta_{\mathcal{D}}\left(X \cap f_{1}\left[B_{1}\right] / X \cap f_{1}[A]\right)+\delta_{\mathcal{D}}\left(X \cap f_{2}\left[B_{2}\right] / X \cap f_{1}[A]\right)
$$

holds whenever $X \subseteq_{\text {fin }} D$, we say that $\mathcal{D}$ is a free amalgam.

We say that $(\mathbb{C}, \delta)$ is a free amalgamation class if whenever $\mathcal{A}, \mathcal{B}_{1}, \mathcal{B}_{2} \in \mathbb{C}$, there exists $\mathcal{D} \in \mathbb{C}$, a free amalgam of $\mathcal{B}_{1}$ and $\mathcal{B}_{2}$ over $\mathcal{A}$.

Since $\emptyset \in \mathbb{C}$, by a considerable generalization of Fraïssé's Theorem, if $\mathbb{C}$ is a free amalgamation class, then there is a unique (up to isomorphism) countable generic structure $\mathcal{M} \in \overline{\mathbb{C}}$ such that

(*) Whenever $\mathcal{A} \leqslant \mathcal{M}$ and $\mathcal{B} \in \mathbb{C}$ is such that $\mathcal{A} \leqslant \mathcal{B}$, there exists a strong embedding $f: \mathcal{B} \rightarrow \mathcal{M}$ fixing $A$ pointwise.

By a standard back and forth argument, every finite partial isomorphism between self-sufficient subsets of countable generic structures for $\mathbb{C}$ extends to a full isomorphism. We unambiguously define the pregeometry associated to $(\mathbb{C}, \delta)$ as the pregeometry associated to its countable generic structure.

We can now explore connections between free amalgamation classes. For the rest of this section, fix $\left(\mathbb{C}_{1}, \delta_{1}\right)$ and $\left(\mathbb{C}_{2}, \delta_{2}\right)$, free amalgamation classes with generic structures $\mathcal{M}_{1}$ and $\mathcal{M}_{2}$, in languages $\mathcal{L}_{1}$ and $\mathcal{L}_{2}$. Structures will be declared as being in the context of either $\left(\mathbb{C}_{1}, \delta_{1}\right)$ or $\left(\mathbb{C}_{2}, \delta_{2}\right)$ directly or indirectly, as described in remarks 2.3 and 2.5. For a structure $\mathcal{A}$, the objects $\delta_{\mathcal{A}}, \mathrm{d}_{\mathcal{A}}, \operatorname{PG}(\mathcal{A})$, and $\mathrm{cl}_{\mathcal{A}}$ are defined with respect to the predimension function associated to $\mathcal{A}$. Similarly, when verbally referring to strong substructures or embeddings, the appropriate notion of self-sufficiency is inferred from the structures involved. When explicitly using mathematical notation, we use $\leqslant_{i}$ to denote self-sufficiency with respect to $\delta_{i}$. 
In the two subsections ahead, we will give sufficient criteria for showing that $\operatorname{PG}\left(\mathcal{M}_{1}\right) \cong \operatorname{PG}\left(\mathcal{M}_{2}\right)$ and for showing that $\mathcal{M}_{2}$ is a definable reduct of $\mathcal{M}_{1}$. Our usage of the term "reduct" is clarified in Definition 2.15.

2.1. Isomorphism of associated pregeometries. The following definition and lemma are a slight strengthening of [EF12, Lemma 2.3].

Definition 2.11. Say that $\mathbb{C}_{1} \stackrel{*}{\rightsquigarrow} \mathbb{C}_{2}$ if, given structures $\mathcal{A}_{i} \in \mathbb{C}_{i}$ such that $\mathrm{PG}\left(\mathcal{A}_{1}\right) \cong \mathrm{PG}\left(\mathcal{A}_{2}\right)$,

- For every $\mathcal{B}_{1} \in \mathbb{C}_{1}$ with $\mathcal{A}_{1} \leqslant 1 \mathcal{B}_{1}$, there exist some $\mathcal{D}_{1} \in \mathbb{C}_{1}$ with $\mathcal{B}_{1} \leqslant{ }_{1} \mathcal{D}_{1}$, and some $\mathcal{D}_{2} \in \mathbb{C}_{2}$ with $\mathcal{A}_{2} \leqslant{ }_{2} \mathcal{D}_{2}$, such that the isomorphism between $\operatorname{PG}\left(\mathcal{A}_{1}\right)$ and $\mathrm{PG}\left(\mathcal{A}_{2}\right)$ can be extended to an isomorphism between $\mathrm{PG}\left(\mathcal{D}_{1}\right)$ and $\mathrm{PG}\left(\mathcal{D}_{2}\right)$.

Lemma 2.12. Assume $\mathbb{C}_{1} \stackrel{*}{\rightsquigarrow} \mathbb{C}_{2}$ and $\mathbb{C}_{2} \stackrel{*}{\rightsquigarrow} \mathbb{C}_{1}$. Let $f_{0}: \operatorname{PG}\left(\mathcal{A}_{1}\right) \rightarrow \operatorname{PG}\left(\mathcal{A}_{2}\right)$ be a finite isomorphism of pregeometries between some $\mathcal{A}_{1} \leqslant_{1} \mathcal{M}_{1}$ and $\mathcal{A}_{2} \leqslant_{2} \mathcal{M}_{2}$. Then $f_{0}$ extends to an isomorphism of pregeometries $f: \mathrm{PG}\left(\mathcal{M}_{1}\right) \rightarrow \operatorname{PG}\left(\mathcal{M}_{2}\right)$.

In particular, building on the empty isomorphism, $\mathrm{PG}\left(\mathcal{M}_{1}\right) \cong \mathrm{PG}\left(\mathcal{M}_{2}\right)$.

Proof. The proof is a standard back and forth between strong substructures. We show the forward direction.

Choose arbitrarily some $x \in \mathcal{M}_{1}$ and let $\mathcal{B}_{1}=\mathcal{M}_{1}\left[\Lambda_{\mathcal{M}_{1}}\left(A_{1} x\right)\right]$, the structure induced on the self-sufficient closure of $A_{1} \cup\{x\}$ in $\mathcal{M}_{1}$. By $\mathbb{C}_{1} \stackrel{*}{\rightsquigarrow} \mathbb{C}_{2}$ there exist some $\mathcal{D}_{1} \in \mathbb{C}_{1}$ with $\mathcal{B}_{1} \leqslant \mathcal{D}_{1}$ and $\mathcal{D}_{2} \in \mathbb{C}_{2}$ with $\mathcal{A}_{2} \leqslant \mathcal{D}_{2}$ such that $f_{0}$ extends to an isomorphism $f \supseteq f_{0}$ between $\mathrm{PG}\left(\mathcal{D}_{1}\right)$ and $\mathrm{PG}\left(\mathcal{D}_{2}\right)$. Using genericity, embed $\mathcal{D}_{1}$ strongly into $\mathcal{M}_{1}$ over $\mathcal{B}_{1}$. Similarly, embed $\mathcal{D}_{2}$ strongly into $\mathcal{M}_{2}$ over $A_{2}$. By renaming elements, we may assume $\mathcal{D}_{i} \leqslant \mathcal{M}_{i}$, hence $\operatorname{PG}\left(\mathcal{D}_{i}\right) \subseteq \operatorname{PG}\left(\mathcal{M}_{i}\right)$. Then we have extended the isomorphism of pregeometries to include $x$ in its domain.

To facilitate the use of Lemma 2.12 down the road, we prove the following.

Lemma 2.13. Let $\mathcal{B}_{1} \in \mathbb{C}_{1}$ and $\mathcal{B}_{2} \in \mathbb{C}_{2}$ have the same universe $B$, and let $A \subseteq B$ be such that $\mathcal{A}_{i} \leqslant_{i} \mathcal{B}_{i}$, where $\mathcal{A}_{i}:=\mathcal{B}_{i}[A]$, for both $i \in\{1,2\}$. Assume that $\operatorname{PG}\left(\mathcal{A}_{1}\right)=\operatorname{PG}\left(\mathcal{A}_{2}\right)$. Then:

(1) If $Y \subseteq B$ is closed in $\mathrm{PG}\left(\mathcal{B}_{1}\right)$, then $\delta_{\mathcal{B}_{1}}(Y \cap A)=\delta_{\mathcal{B}_{2}}(Y \cap A)$

(2) If $Y \subseteq B$ is closed in $\mathrm{PG}\left(\mathcal{B}_{1}\right)$ and $\delta_{\mathcal{B}_{1}}(Y / Y \cap A) \geq \delta_{\mathcal{B}_{2}}(Y / Y \cap A)$, then for any $X \subseteq B$ such that $\operatorname{cl}_{\mathcal{B}_{1}}(X)=Y$, it holds that $\mathrm{d}_{\mathcal{B}_{1}}(X) \geq \mathrm{d}_{\mathcal{B}_{2}}(X)$.

(3) If $\delta_{\mathcal{B}_{1}}(Y / Y \cap A)=\delta_{\mathcal{B}_{2}}(Y / Y \cap A)$ for every $Y \subseteq B$ closed in either $\operatorname{PG}\left(\mathcal{B}_{1}\right)$ or $\mathrm{PG}\left(\mathcal{B}_{2}\right)$, then $\mathrm{PG}\left(\mathcal{B}_{1}\right)=\operatorname{PG}\left(\mathcal{B}_{2}\right)$.

Proof. Recall that $\mathcal{A}_{i} \leqslant i \mathcal{B}_{i}$ implies $\operatorname{PG}\left(\mathcal{A}_{i}\right) \subseteq \operatorname{PG}\left(\mathcal{B}_{i}\right)$.

(1) If $Y$ is closed in $\operatorname{PG}\left(\mathcal{B}_{1}\right)$, then $Y \cap A$ is closed in $\operatorname{PG}\left(\mathcal{A}_{1}\right)$. By assumption $\mathrm{PG}\left(\mathcal{A}_{1}\right)=\operatorname{PG}\left(\mathcal{A}_{2}\right)$, so $Y \cap A$ is also closed in $\mathcal{A}_{2}$ and $\mathrm{d}_{\mathcal{A}_{1}}(Y \cap A)=\mathrm{d}_{\mathcal{A}_{2}}(Y \cap$ $A)$. A closed set is self-sufficient, $\operatorname{so~}_{\mathcal{A}_{i}}(Y \cap A)=\delta_{\mathcal{A}_{i}}(Y \cap A)=\delta_{\mathcal{B}_{i}}(Y \cap A)$.

(2) Let $X \subseteq Y$ be such that $\operatorname{cl}_{\mathcal{B}_{1}}(X)=Y$, then $\mathrm{d}_{\mathcal{B}_{1}}(X)=\mathrm{d}_{\mathcal{B}_{1}}(Y)$. Since $Y$ is closed in $\mathcal{B}_{1}$, it is self-sufficient in $\mathcal{B}_{1}$, so $\mathrm{d}_{\mathcal{B}_{1}}(Y)=\delta_{\mathcal{B}_{1}}(Y)$. By $(1)$ and the assumption $\delta_{\mathcal{B}_{1}}(Y / Y \cap A) \geq \delta_{\mathcal{B}_{2}}(Y / Y \cap A)$, we have $\delta_{\mathcal{B}_{1}}(Y) \geq \delta_{\mathcal{B}_{2}}(Y)$. By definition of dimension and $X \subseteq Y$, we have $\delta_{\mathcal{B}_{2}}(Y) \geq \mathrm{d}_{\mathcal{B}_{2}}(Y) \geq \mathrm{d}_{\mathcal{B}_{2}}(X)$.

(3) By the previous item, $\mathrm{d}_{\mathcal{B}_{1}}(X)=\mathrm{d}_{\mathcal{B}_{2}}(X)$ for every $X \subseteq B$. 
Remark 2.14. In the proof of (2) and (3) of Lemma 2.13 above, one may replace the assumption that $\mathcal{A}_{i} \leqslant \mathcal{B}_{i}$ by the consequence of (1). In particular, (2) and (3) hold when $\delta_{\mathcal{A}_{1}}=\delta_{\mathcal{A}_{2}}$.

2.2. Reduction. In this paper, "definable reduct" will be used in the precise sense of Definition 2.15. Intuitively, every definable set in the reduct $\mathcal{N}_{r}$ is definable in the "original" structure $\mathcal{N}$, but not necessarily vice versa.

Definition 2.15. Given two first-order languages $\mathcal{L}$ and $\mathcal{L}_{r}$, for an $\mathcal{L}$-structure $\mathcal{N}$ and an $\mathcal{L}_{r}$-structure $\mathcal{N}_{r}$ with the same universe as $\mathcal{N}$, we say that $\mathcal{N}_{r}$ is a definable reduct of $\mathcal{N}$ if for every symbol $S \in \mathcal{L}_{r}$ there is an $\mathcal{L}$-formula $\varphi_{S}$ (perhaps with parameters from $\mathcal{N}$ ) such that $\varphi_{S}(\mathcal{N})=S^{\mathcal{N}_{r}}$. Given such a choice of formulas, we say that $\mathcal{M}_{r}$ is the reduct of $\mathcal{M}$ to $\left\{\varphi_{S}: S \in \mathcal{L}\right\}$.

If, in addition, $\mathcal{N}$ is not a definable reduct of $\mathcal{N}_{r}$, we say that $\mathcal{N}_{r}$ is a proper definable reduct of $\mathcal{N}$.

Variations on the definition above include replacing $\varphi_{S}$ with a type (type-definable reduct), an infinite disjunction ( $\vee$-definable reduct), and so on.

We return to establishing a criterion for $\mathcal{M}_{2}$ being a (proper) definable reduct of $\mathcal{M}_{1}$. As is apparent from the definition of a definable reduct, here $\mathbb{C}_{1}$ and $\mathbb{C}_{2}$ do not play a symmetric role. Properties P1-P3 of the following assumption guarantee that $\mathcal{M}_{2}$ is indeed the "reduct" of $\mathcal{M}_{1}$ given by $\widehat{\mathcal{M}}_{1}$ (defined therein), whereas $\mathrm{P} 4$ only matters to the irreversibility of the "reduction".

Assumption 2.16. To each $\mathcal{L}_{1}$-structure $\mathcal{A}$ with universe $A$, associate an $\mathcal{L}_{2^{-}}$ structure $\widehat{\mathcal{A}}$ with the same universe, so that the association is invariant under isomorphism, i.e., $\mathcal{A} \cong \mathcal{B} \Longrightarrow \widehat{\mathcal{A}} \cong \widehat{\mathcal{B}}$, under the same bijection. ${ }^{2}$

We assume the following properties of $\wedge, \mathbb{C}_{1}$, and $\mathbb{C}_{2}$ :

P1. Whenever $\mathcal{A} \leqslant_{1} \mathcal{N} \in \overline{\mathbb{C}}_{1}$ with $A$ finite, then $\widehat{\mathcal{A}}=\widehat{\mathcal{N}}[A]$.

P2. If $\mathcal{A} \in \mathbb{C}_{1}$, then $\widehat{\mathcal{A}} \in \mathbb{C}_{2}$.

P3. Whenever $\mathcal{A} \in \mathbb{C}_{1}, \mathcal{B} \in \mathbb{C}_{2}$ are such that $\widehat{\mathcal{A}} \leqslant 2 \mathcal{B}$, then there exists some $\mathcal{E} \in \mathbb{C}_{1}$ with $\mathcal{A} \leqslant_{1} \mathcal{E}$ and $\mathcal{B} \leqslant_{2} \widehat{\mathcal{E}}$.

P4. For any $\mathcal{F} \in \mathbb{C}_{1}$, there exist $\mathcal{A}, \mathcal{B} \in \mathbb{C}_{1}$ with $\mathcal{F} \leqslant 1 \mathcal{A}, \mathcal{B}$, and $f: A \rightarrow B$ fixing $F$ pointwise such that $f$ is an isomorphism between $\widehat{\mathcal{A}}$ and $\widehat{\mathcal{B}}$, but not an isomorphism between $\mathcal{A}$ and $\mathcal{B}$.

We will show with a series of short claims that these conditions are sufficient so that $\widehat{\mathcal{M}}_{1} \cong \mathcal{M}_{2}$ (i.e., $\mathcal{M}_{2}$ is a proper definable reduct of $\left.\mathcal{M}_{1}\right)$. And that $\operatorname{Aut}\left(\mathcal{M}_{1}\right) \subset$ $\operatorname{Aut}\left(\widehat{\mathcal{M}}_{1}\right)$, where $\operatorname{Aut}(\mathcal{N})$ denotes the automorphism group of a structure $\mathcal{N}$. We indicate in each lemma which properties of Assumption 2.16 it requires.

Lemma 2.17 (P1-P2). If $\mathcal{N} \in \overline{\mathbb{C}}_{1}$, then $\widehat{\mathcal{N}} \in \overline{\mathbb{C}}_{2}$.

Proof. Let $X \subseteq_{\text {fin }} N$ be arbitrary. Denote $A=\Lambda_{\mathcal{N}}(X)$ and $\mathcal{A}=\mathcal{N}[A]$. P1 implies that $\widehat{\mathcal{N}}[A]=\widehat{\mathcal{A}}$ and P2 implies that $\widehat{\mathcal{A}} \in \mathbb{C}_{2}$. Since $\mathbb{C}_{2}$ is closed under taking substructures and $\widehat{\mathcal{N}}[X] \subseteq \widehat{\mathcal{N}}[A]$, we get $\widehat{\mathcal{N}}[X] \in \mathbb{C}_{2}$. We chose $X$ arbitrarily, so $\widehat{\mathcal{N}} \in \overline{\mathbb{C}}_{2}$.

\footnotetext{
${ }^{2}$ Later in this paper, the association will be by taking a definable reduct, but the following is applicable also to type-definable reducts, $\bigvee$-definable reducts, or any other arbitrary map satisfying Assumption 2.16.
} 
Lemma $2.18(\mathrm{P} 1-\mathrm{P} 2)$. If $\mathcal{A} \leqslant 1 \mathcal{B} \in \mathbb{C}_{1}$, then $\widehat{\mathcal{A}} \leqslant 2 \widehat{\mathcal{B}}$.

Proof. By $\mathrm{P} 1, \widehat{\mathcal{B}}[A]=\widehat{\mathcal{A}}$. It suffices to show that $\delta_{2}(\widehat{\mathcal{B}} / \widehat{\mathcal{A}}) \geq 0$.

For a natural $r$, let $\mathcal{D} \in \mathbb{C}_{1}$ be a free amalgam of $\mathcal{B}_{1}, \ldots, \mathcal{B}_{r}$ - distinct copies of $\mathcal{B}$ over $\mathcal{A}$-over $\mathcal{A}$. Then, up to renaming elements of $D, \mathcal{A}, \mathcal{B}_{1}, \ldots, \mathcal{B}_{r} \leqslant 1$ D. By $\mathrm{P} 1, \widehat{\mathcal{A}}=\widehat{D}[A]$, and $\widehat{\mathcal{B}}_{i}=\widehat{\mathcal{D}}\left[B_{i}\right]$ for each $i \leq r$. Iterating submodularity, we have

$$
\delta_{2}(\widehat{\mathcal{D}} / \widehat{\mathcal{A}}) \leq r \cdot \delta_{2}(\widehat{\mathcal{B}} / \widehat{\mathcal{A}}) .
$$

By $\mathrm{P} 2, \widehat{\mathcal{D}} \in \mathbb{C}_{2}$, hence $\delta_{2}(\widehat{\mathcal{D}}) \geq 0$, so $\delta_{2}(\widehat{\mathcal{D}} / \widehat{\mathcal{A}}) \geq-\delta_{2}(\widehat{\mathcal{A}})$. As $r$ can be chosen arbitrarily large, $\delta_{2}(\widehat{\mathcal{B}} / \widehat{\mathcal{A}})$ must be non-negative.

We remind the reader of Remark 2.5 prior to the next proof, which lifts Lemma 2.18 to countable structures.

Corollary 2.19 (P1-P2). If $\mathcal{P} \leqslant_{1} \mathcal{N} \in \overline{\mathbb{C}}_{1}$, then $\widehat{\mathcal{P}} \leqslant{ }_{2} \widehat{\mathcal{N}}$.

Proof. Assume $\mathcal{P} \leqslant 1 \mathcal{N}$ and let $X \subseteq_{\text {fin }} N$. Take $B=\Lambda_{\mathcal{N}}(X)$ and $\mathcal{B}=\mathcal{N}[B]$. As $P \leqslant_{1} \mathcal{N}$, by submodularity, $B \cap P \leqslant_{1} \mathcal{B}$. Then by Lemma 2.18 we have $B \cap P \leqslant_{2} \widehat{\mathcal{B}}$, hence $\delta_{\widehat{\mathcal{B}}}(X / X \cap P) \geq 0$. By P1, $\widehat{\mathcal{B}}=\widehat{\mathcal{N}}[B]$, so $\delta_{\widehat{\mathcal{N}}}(X / X \cap P) \geq 0$. Thus, $P \leqslant{ }_{2} \widehat{\mathcal{N}}$.

To see $\widehat{\mathcal{N}}[P]=\widehat{\mathcal{P}}$ note that for any $\mathcal{A} \leqslant_{1} \mathcal{P}$, P1 implies $\widehat{\mathcal{P}}[A]=\widehat{\mathcal{A}}=\widehat{\mathcal{N}}[A]$.

We use the defining property of a generic structure for $\mathbb{C}_{2}$ to attain the result of Proposition 2.20. See the extension property $(*)$ described in the discussion following Definition 2.10 for a reminder regarding generic structures. Recall that $\mathcal{M}_{1}$ and $\mathcal{M}_{2}$ are generic.

Proposition 2.20 (P1-P3). $\widehat{\mathcal{M}}_{1} \cong \mathcal{M}_{2}$.

Proof. We need to show that $\widehat{\mathcal{M}}_{1}$ is generic for $\mathbb{C}_{2}$. We know that $\widehat{\mathcal{M}}_{1} \in \overline{\mathbb{C}}_{2}$ by Lemma 2.17 , so we only need to show that $\widehat{\mathcal{M}}_{1}$ has the extension property $(*)$ with respect to $\mathbb{C}_{2}$.

Suppose $\mathcal{A} \leqslant_{2} \widehat{\mathcal{M}}_{1}$ and $\mathcal{B} \in \mathbb{C}_{2}$ is such that $\mathcal{A} \leqslant_{2} \mathcal{B}$. Denote the universe of $\mathcal{A}$ by $A$, let $C=\Lambda_{\mathcal{M}_{1}}(A)$, and denote $\mathcal{C}=\mathcal{M}_{1}[C]$. Then P1 implies $\widehat{\mathcal{C}}=\widehat{\mathcal{M}}_{1}[C], \mathrm{P} 2$ implies $\widehat{\mathcal{C}} \in \overline{\mathbb{C}}_{2}$, and by $\mathcal{A} \leqslant_{2} \widehat{\mathcal{M}}_{1}$ we have $\mathcal{A} \leqslant_{2} \widehat{\mathcal{C}}$. Let $\mathcal{D} \in \mathbb{C}_{2}$ be an amalgam of $\widehat{\mathcal{C}}$ and $\mathcal{B}$ over $\mathcal{A}$. We may assume $\widehat{\mathcal{C}} \leqslant_{2} \mathcal{D}$.

By P3, choose $\mathcal{E} \in \mathbb{C}_{1}$ with $\mathcal{C} \leqslant_{1} \mathcal{E}$ and $\mathcal{D} \leqslant_{2} \widehat{\mathcal{E}}$. As $\mathcal{M}_{1}$ is generic for $\mathbb{C}_{1}$ and $\mathcal{C} \leqslant_{1} \mathcal{M}_{1}$, we may assume $\mathcal{C} \leqslant_{1} \mathcal{E} \leqslant_{1} \mathcal{M}_{1}$. By Corollary 2.19, $\widehat{\mathcal{E}} \leqslant_{2} \widehat{\mathcal{M}}_{1}$. By construction, $\mathcal{A} \leqslant_{2} \widehat{\mathcal{C}} \leqslant_{2} \mathcal{D} \leqslant_{2} \widehat{\mathcal{E}}$ and $\mathcal{B}$ can be strongly embedded into $\mathcal{D}$ over $\mathcal{A}$. In particular, we have found a strong embedding of $\mathcal{B}$ into $\widehat{\mathcal{M}}_{1}$ over $\mathcal{A}$.

For a structure $\mathcal{N}$ and a set $F \subseteq N$, denote by $\operatorname{Aut}_{F}(\mathcal{N})$ the group of automorphisms of $\mathcal{N}$ fixing $F$ pointwise.

Proposition $2.21(\mathrm{P} 1-\mathrm{P} 4)$. For any finite $F \subseteq M_{1}, \operatorname{Aut}_{F}\left(\mathcal{M}_{1}\right) \subset \operatorname{Aut}_{F}\left(\widehat{\mathcal{M}}_{1}\right)$. In particular, $\operatorname{Aut}_{F}\left(\mathcal{M}_{1}\right) \neq \operatorname{Aut}_{F}\left(\widehat{\mathcal{M}}_{1}\right)$.

Proof. To see $\operatorname{Aut}_{F}\left(\mathcal{M}_{1}\right) \subseteq \operatorname{Aut}_{F}\left(\widehat{\mathcal{M}}_{1}\right)$, let $\sigma \in \operatorname{Aut}_{F}\left(\mathcal{M}_{1}\right)$, let $X \subseteq$ fin $M_{1}$ and denote $Y=\sigma[X]$. Without loss of generality, by extending $X$, we may assume $X \leqslant_{1} \mathcal{M}_{1}$, and therefore $Y \leqslant_{1} \mathcal{M}_{1}$. Then by $\left.\mathrm{P} 1, \widehat{\mathcal{M}}_{1}[X]=\widehat{\mathcal{M}_{1}[X]} \cong \widehat{\mathcal{M}_{1}[Y}\right]=$ $\widehat{\mathcal{M}}_{1}[Y]$, meaning $\sigma$ is also an automorphism of $\widehat{\mathcal{M}}_{1}$.

Now we show inequality of the automorphism groups. By extending to its selfsufficient closure, assume $F \leqslant_{1} \mathcal{M}_{1}$. Let $\mathcal{A}, \mathcal{B} \in \mathbb{C}_{1}$ and $f: A \rightarrow B$ be as guaranteed 
by $\mathrm{P} 4$. Since $\mathcal{M}_{1}$ is generic for $\mathbb{C}_{1}$, we may assume $\mathcal{A}, \mathcal{B} \leqslant_{1} M_{1}$. By Corollary 2.19, we have $\widehat{\mathcal{A}}, \widehat{\mathcal{B}} \leqslant 2 \widehat{\mathcal{M}}_{1}$. By Proposition $2.20, \widehat{\mathcal{M}}_{1}$ is a generic structure for $\mathbb{C}_{2}$. So $f$ extends to an automorphism of $\widehat{\mathcal{M}}_{1}$, which is not an automorphism of $\mathcal{M}_{1}$.

Corollary 2.22. If $\mathcal{L}_{1}$ is finite and the map $\mathcal{N} \mapsto \widehat{\mathcal{N}}$ is the operation of taking a definable reduct, then $\widehat{\mathcal{M}}_{1}$ is a proper reduct, i.e., $\mathcal{M}_{1}$ is not interdefinable with $\widehat{\mathcal{M}}_{1}$.

Proof. Had $\mathcal{M}_{1}$ been interdefinable with $\widehat{\mathcal{M}}_{1}$, letting $F$ be the (finite) set of parameters required to define $\mathcal{M}_{1}$ in $\widehat{\mathcal{M}}_{1}$, any automorphism of $\widehat{\mathcal{M}}_{1}$ fixing $F$ is also an automorphism of $M_{1}$, contradicting Proposition 2.21.

\section{VARYing SYMmetry in HRUSHOVSKI'S NON-COLLAPSED CONSTRUCTION}

Fix some natural $n \geq 3$. We denote by $S_{n}$ the group of permutations of $n$ elements, under the operation of composition. The elements $\sigma \in S_{n}$ act on the space of $n$-tuples by $\sigma\left(a_{1}, \ldots, a_{n}\right)=\left(a_{\sigma(1)}, \ldots, a_{\sigma(n)}\right)$.

Definition 3.1. For an $n$-ary relation $R$ and a subgroup $\mathfrak{g} \leq S_{n}$, we say that

- $R$ is irreflexive if whenever $\left(a_{1}, \ldots, a_{n}\right) \in R$, then the elements $a_{1}, \ldots, a_{n}$ are pairwise distinct.

- $R$ is $\mathfrak{g}$-symmetric if $\mathfrak{g} \cdot R=R$, i.e., whenever $\left(a_{1}, \ldots, a_{n}\right) \in R$, then $\left\{\sigma\left(a_{1}, \ldots, a_{n}\right): \sigma \in \mathfrak{g}\right\} \subseteq R$.

We describe for each $\mathfrak{g} \leq S_{n}$ a generic structure $\mathcal{M}_{\mathfrak{g}}$. In the spirit of Remark 2.3, we let $\left\{\mathcal{L}_{\mathfrak{g}}: \mathfrak{g} \leq S_{n}\right\}$ be a collection of disjoint languages, where $\mathcal{L}_{\mathfrak{g}}$ is the language of a single $n$-ary relation symbol $R_{\mathfrak{g}}$. From now on, we restrict our discussion of $\mathcal{L}_{\mathfrak{g}^{-}}$ structures only to those structures $\mathcal{A}$ such that $R_{\mathfrak{g}}^{\mathcal{A}}$ is irreflexive and $\mathfrak{g}$-symmetric (i.e., the class $\mathbb{C}_{0}^{\mathfrak{g}}$ as defined in Definition 3.5). Before proceeding, we introduce some important notation and naming conventions, motivated by the predimension function associated to $\mathcal{L}_{\mathfrak{g}}$-structures (Definition 3.6).

Notation 3.2. For a subgroup $\mathfrak{g} \leq S_{n}$, we denote the orbit of a tuple $\left(a_{1}, \ldots, a_{n}\right)$ under $\mathfrak{g}$ by $\left[a_{1}, \ldots, a_{n}\right]_{\mathfrak{g}}=\left\{\sigma\left(a_{1}, \ldots, a_{n}\right): \sigma \in \mathfrak{g}\right\}$. We will sometimes refer to such an orbit as an edge or, more specifically, a $\mathfrak{g}$-symmetric edge. We use symmetric edge, omitting $\mathfrak{g}$, when $\mathfrak{g}$ is the full group $S_{n}$.

For a relation $R$, abusing notation, we write $\left[a_{1}, \ldots, a_{n}\right]_{\mathfrak{g}} \in R$ to mean $\left[a_{1}, \ldots, a_{n}\right]_{\mathfrak{g}} \subseteq$ $R$.

Remark 3.3. To avoid ambiguity in formalism, the object $R_{\mathfrak{g}}^{\mathcal{A}}$ is a set of ordered tuples, in accordance with standard notation. At times, we may wish to think of it as a set of $\mathfrak{g}$-orbits (recall that $\mathfrak{g}$ permutes elements within a single tuple, rather than permuting tuples in a structure). Thus, when defining a structure $\mathcal{A}$, often we will let $G$ be a set of $\mathfrak{g}$-orbits, and take $R_{\mathfrak{g}}^{\mathcal{A}}$ to be $\bigcup G$. This difference is why later, in Definition 3.20, we define both $Q(a ; b)$ and $G(a ; b)$.

Notation 3.4. The full and trivial subgroups, $S_{n}$ and $\{\mathrm{id}\}$, respectively, will be of special importance to us. We write $\sim$ for the full subgroup $S_{n}$, and $\nsim$ for the trivial subgroup $\{\mathrm{id}\}$. For example, $\mathcal{C}_{\nsim}, \delta_{\sim}, \mathcal{L}_{\nsim}$, etc..

We also use special notation for an orbit under the full subgroup. Instead of $\left[a_{1}, \ldots, a_{n}\right]_{\sim}$, we write $\left[a_{1}, \ldots, a_{n}\right]$, omitting the $\sim$ subscript.

As for the special subgroup $\{\mathrm{id}\}$, since an orbit is just a single tuple, the notation $\left[a_{1}, \ldots, a_{n}\right]_{\nsim}$ will never be invoked. 
Returning to $\mathcal{M}_{\mathfrak{g}}$, to construct the structure we only need to define the class $\mathcal{C}_{0}^{\mathfrak{g}}$, a predimension function $\delta_{\mathfrak{g}}$, and follow the procedure presented in the first part of Section 2.

Definition 3.5. For each $\mathfrak{g} \leq S_{n}$, define $\mathcal{C}_{0}^{\mathfrak{g}}$ to be the class of finite irreflexive ${ }^{3}$ $\mathfrak{g}$-symmetric $\mathcal{L}_{\mathfrak{g}}$-structures.

The predimension used for Hrushovski's construction assigns to a structure $\mathcal{A}$ the difference between the cardinality of $A$ and the number of instances of the relation $R$ in $\mathcal{A}$. In the case of a $\mathfrak{g}$-symmetric structure, the natural generalization is to count an entire $\mathfrak{g}$-orbit as a single "instance".

Definition 3.6. For each $\mathcal{A} \in \mathcal{C}_{0}^{\mathfrak{g}}$ define

$$
\delta_{\mathfrak{g}}(\mathcal{A})=|A|-\left|\left\{\left[a_{1}, \ldots, a_{n}\right]_{\mathfrak{g}}:\left(a_{1}, \ldots, a_{n}\right) \in R_{\mathfrak{g}}^{\mathcal{A}}\right\}\right|,
$$

the number of points in $\mathcal{A}$ minus the number of $\mathfrak{g}$-orbits contained in $R_{\mathfrak{g}}^{\mathcal{A}}$.

Each function $\delta_{\mathfrak{g}}$ is shown to be submodular by using the inclusion-exclusion principle, and is hence a predimension function for $\mathcal{C}_{0}^{\mathfrak{g}}$ (see Definition 2.1). We associate to $\delta_{\mathfrak{g}}$ (and to $\mathcal{C}_{0}^{\mathfrak{g}}$ ) the notion of self-sufficiency $\leqslant_{\mathfrak{g}}$, and similarly define $\leqslant \sim$ and $\leqslant x$.

Observation 3.7. Were we to identify $\mathcal{L}_{\nsim}, \mathcal{L}_{\sim}$ and some $\mathcal{L}_{\mathfrak{g}}$ with each other, examining a structure $\mathcal{A}$ yields $\delta_{\nsim}(\mathcal{A}) \leq \delta_{\mathfrak{g}}(\mathcal{A}) \leq \delta_{\sim}(\mathcal{A})$. Consequently, again up to identifying the languages, for some $\mathcal{B} \supseteq \mathcal{A}$, we have $\mathcal{A} \leqslant_{\chi} \mathcal{B} \Longrightarrow \mathcal{A} \leqslant_{\mathfrak{g}} \mathcal{B} \Longrightarrow$ $\mathcal{A} \leqslant \sim \mathcal{B}$.

Formally, by "identifying languages", we mean considering $(A, R)$, where $A$ is a set and $R \subseteq A^{n}$, once as an $\mathcal{L}_{\chi}$-structure, once as an $\mathcal{L}_{\mathfrak{g}}$-structure, and once as an $\mathcal{L}_{\sim}$-structure. In the paragraph above, despite denoting all three structures by $\mathcal{A}$, we trust the reader to determine which $\mathcal{A}$ is in what language.

The stage is set to define $\mathcal{C}_{\mathfrak{g}}$ from $\mathcal{C}_{0}^{\mathfrak{g}}$. Note that in light of the observation above, up to identifying languages and foregoing symmetry assumptions, $\mathcal{C}_{\nsim} \subset \mathcal{C}_{\mathfrak{g}} \subset \mathcal{C}_{\sim}$.

Definition 3.8. For each $\mathfrak{g} \leq S_{n}$, define $\mathcal{C}_{\mathfrak{g}}=\left\{\mathcal{A} \in \mathcal{C}_{0}^{\mathfrak{g}}: \emptyset \leqslant \mathfrak{g} \mathcal{A}\right\}$.

The classes defined above are free amalgamation classes with respect to their respective predimension functions. The class $\mathcal{C}_{\nsim}$ gives rise to the (non-collapsed) construction of [Hru93]. We denote by $\mathcal{M}_{\mathfrak{g}}$ the generic structures associated to $\mathcal{C}_{\mathfrak{g}}$. We remind the reader of the special notation $\mathcal{M}_{\nsim}$ and $\mathcal{M}_{\sim}$. Using the methods of subsections 2.1 and 2.2 , we will show that

Theorem 3.9. Whenever $\mathfrak{g}, \mathfrak{h} \leq S_{n}$, then $\mathrm{PG}\left(\mathcal{M}_{\mathfrak{g}}\right) \cong \operatorname{PG}\left(\mathcal{M}_{\mathfrak{h}}\right)$ and $\mathcal{M}_{\mathfrak{h}}$ is isomorphic to a proper definable reduct of $\mathcal{M}_{\mathfrak{g}}$.

\footnotetext{
${ }^{3}$ In his paper, Hrushovski did not require irreflexivity of the relation, but it is easy to take a reduct of the original construction which preserves only irreflexive tuples, making the relation irreflexive. The question of irreflexivity also does not affect the isomorphism type of the associated pregeometry. Thus, for the purpose of this paper, there is no harm in assuming all structures are irreflexive.
} 
3.1. Pregeometries. For this subsection we fix some $\mathfrak{g} \leq S_{n}$. We will show that $\mathrm{PG}\left(\mathcal{M}_{\mathfrak{g}}\right) \cong \mathrm{PG}\left(\mathcal{M}_{\nsim}\right)$ using Lemma 2.12 , building on the empty isomorphism. Since $\mathfrak{g}$ is arbitrary, this proves the first part of Theorem 3.9.

Recall Definition 2.11. Given the definitions of $\delta_{\mathfrak{g}}$ and $\delta_{\nsim}$, it is easy to show $\mathcal{C}_{\mathfrak{g}} \stackrel{*}{\rightsquigarrow} \mathcal{C}_{\nsim}$. We merely need to replace every $\mathfrak{g}$-orbit in a $\mathfrak{g}$-symmetric extension with a single representative tuple.

Lemma 3.10. $\mathcal{C}_{\mathfrak{g}} \stackrel{*}{\rightsquigarrow} \mathcal{C}_{\nsim}$

Proof. Let $\mathcal{A}_{1} \in \mathcal{C}_{\mathfrak{g}}$ and $\mathcal{A}_{2} \in \mathcal{C}_{\nsim}$ be such that $\mathrm{PG}\left(\mathcal{A}_{1}\right) \cong \mathrm{PG}\left(\mathcal{A}_{2}\right)$. We may assume $\mathcal{A}_{1}$ and $\mathcal{A}_{2}$ have the same universe $A$, and so $\mathrm{PG}\left(\mathcal{A}_{1}\right)=\operatorname{PG}\left(\mathcal{A}_{2}\right)$. Let $\mathcal{B}_{1} \in \mathcal{C}_{\mathfrak{g}}$ with $\mathcal{A}_{1} \leqslant \mathfrak{g} \mathcal{B}_{1}$.

Define on $R_{\mathfrak{g}}^{\mathcal{B}_{1}} \backslash R_{\mathfrak{g}}^{\mathcal{A}_{1}}$ the equivalence relation $\equiv_{\mathfrak{g}}$, where $\bar{x} \equiv_{\mathfrak{g}} \bar{y}$ if and only if $\bar{y} \in[\bar{x}]_{\mathfrak{g}}$. Let $R_{2}$ be a set of representatives for the equivalence classes of $\equiv_{\mathfrak{g}}$, and define $\mathcal{B}_{2}$ to be the structure in $\mathcal{C}_{0}^{\nsim}$ with the same universe as $\mathcal{B}_{1}$ and $R_{\nsim}^{\mathcal{B}_{2}}=R_{\nsim}^{\mathcal{A}_{2}} \cup R_{2}$. Observe that for every subset $Y$ of the universe of $\mathcal{B}_{1}$, we have $\delta_{\mathcal{B}_{1}}(Y / Y \cap A)=$ $\delta_{\mathcal{B}_{2}}(Y / Y \cap A)$, so Lemma 2.13 gives $\operatorname{PG}\left(\mathcal{B}_{1}\right)=\operatorname{PG}\left(\mathcal{B}_{2}\right)$.

In the other direction, to get $\mathcal{C}_{\chi} \stackrel{*}{\rightsquigarrow} \mathcal{C}_{\mathfrak{g}}$, what we would like to do is replace every $R_{\nsim}$-related tuple with its $\mathfrak{g}$-orbit. However, this mapping need not be injective if there is more than one instance of the relation on the same tuple. Our strategy is to extend the structure to one where this never occurs, while preserving the pregeometry, and only then "symmetrize" each related tuple.

Definition 3.11. Let $\mathcal{A}, \mathcal{B} \in \mathcal{C}_{\nsim}$ with $\mathcal{A} \leqslant \nsim \mathcal{B}$. For each $\bar{a}:=\left(a_{1}, \ldots, a_{n}\right) \in$ $R_{\nsim}^{\mathcal{B}} \backslash R_{\nsim}^{\mathcal{A}}$, let $e^{\bar{a}}$ be a new element. Define $\mathcal{D}[\mathcal{B} / \mathcal{A}]$ and $\check{\mathcal{D}}[\mathcal{B} / \mathcal{A}]$ to be the structures in $\mathcal{C}_{0}^{\chi}$ with universe $D:=B \cup\left\{e^{\bar{a}}: \bar{a} \in R_{\nsim}^{\mathcal{B}} \backslash R_{\nsim}^{\mathcal{A}}\right\}$ and

$$
\begin{gathered}
R_{\nsim}^{\mathcal{D}[\mathcal{B} / \mathcal{A}]}=R_{\nsim}^{\mathcal{B}} \cup\left\{\left(a_{1}, \ldots, a_{n-1}, e^{\bar{a}}\right): \bar{a} \in R_{\nsim}^{\mathcal{B}} \backslash R_{\not}^{\mathcal{A}}\right\} \\
R_{\nsim}^{\check{\mathcal{D}}[\mathcal{B} / \mathcal{A}]}=R_{\nsim}^{\mathcal{A}} \cup\left\{\left(a_{1}, \ldots, a_{n-1}, e^{\bar{a}}\right),\left(a_{2}, \ldots, a_{n}, e^{\bar{a}}\right): \bar{a} \in R_{\nsim}^{\mathcal{B}} \backslash R_{\nsim}^{\mathcal{A}}\right\}
\end{gathered}
$$

Intuitively, in both $\mathcal{D}[\mathcal{B} / \mathcal{A}]$ and $\check{\mathcal{D}}[\mathcal{B} / \mathcal{A}]$, we "replace" each related $n$-tuple $\bar{a}$ with a "doubly-related" set of size $n+1$, composed of the $n$ elements of the tuple $\bar{a}$ and the new element $e^{\bar{a}}$. To clarify, here "doubly-related" means that on the $n+1$ elements of the set, there are two distinct related $n$-tuples. While the isomorphism type of such an $n+1$-sized set differs between the structures, this is indistinguishable geometrically.

Lemma 3.12. In the notation of Definition 3.11, denote $\mathcal{D}:=\mathcal{D}[\mathcal{B} / \mathcal{A}]$ and $\check{\mathcal{D}}:=$ $\check{\mathcal{D}}[\mathcal{B} / \mathcal{A}]$. Then $\mathcal{A} \leqslant \nsim \mathcal{B} \leqslant \nsim \mathcal{D}, \mathcal{A} \leqslant \nsim$ D and $\operatorname{PG}(\mathcal{D})=\operatorname{PG}(\check{\mathcal{D}})$.

Proof. Say that a set $X \subseteq D$ is good if whenever $\bar{a}:=\left(a_{1}, \ldots, a_{n}\right) \in R_{\nsim}^{\mathcal{B}} \backslash R_{\chi}^{\mathcal{A}}$ is such that $\left|X \cap\left\{e^{\bar{a}}, a_{1}, \ldots, a_{n}\right\}\right| \geq n-1$, then $\left\{e^{\bar{a}}, a_{1}, \ldots, a_{n}\right\} \subseteq X$. For $X$ a good set, $\left|R_{\nsim}^{\mathcal{D}} \cap X^{n}\right|=\left|R_{\nsim}^{\check{\mathcal{D}}} \cap X^{n}\right|$, so $\delta_{\mathcal{D}}(X)=\delta_{\breve{\mathcal{D}}}(X)$. If $X$ is closed, either in $\operatorname{PG}(\mathcal{D})$ or in $\operatorname{PG}(\check{\mathcal{D}})$, it is a good set, hence $\delta_{\mathcal{D}}(X)=\delta_{\breve{\mathcal{D}}}(X)$. Recalling that $\delta_{\mathcal{D}}(X \cap A)=\delta_{\mathcal{A}}(X \cap A)=\delta_{\check{\mathcal{D}}}(X \cap A)$, by Lemma 2.13 and its following remark, $\operatorname{PG}(\mathcal{D})=\operatorname{PG}(\tilde{\mathcal{D}})$.

Clearly $\mathcal{B} \leqslant \nsim \mathcal{D}$, since the addition of $e^{\bar{a}}$ to any set introduces at most one new related tuple. By transitivity, $\mathcal{A} \leqslant \nsim \mathcal{D}$, hence $\mathrm{d}_{\mathcal{D}}(A)=\delta_{\nsim}(\mathcal{A})$. As $\mathrm{d}_{\mathcal{D}}(A)=$ $\mathrm{d}_{\mathcal{D}}(A)=\delta_{\nsim}(\mathcal{A})$, we get also $\mathcal{A} \leqslant \nsim$ $\check{\mathcal{D}}$. 
By construction, a simple "symmetrization" of $\check{\mathcal{D}}[\mathcal{B} / \mathcal{A}]$ preserves the predimension function, hence the pregeometry. Thus, we are able now to parallel $\mathcal{D}[\mathcal{B} / \mathcal{A}]$ with a structure in $\mathcal{C}_{\mathfrak{g}}$ by passing through $\check{\mathcal{D}}[\mathcal{B} / \mathcal{A}]$.

Lemma 3.13. $\mathcal{C}_{\nsim} \stackrel{*}{\rightsquigarrow} \mathcal{C}_{\mathfrak{g}}$.

Proof. Let $\mathcal{A}_{1} \in \mathcal{C}_{\chi}, \mathcal{A}_{2} \in \mathcal{C}_{\mathfrak{g}}$ with common universe $A$ be such that $\operatorname{PG}\left(\mathcal{A}_{1}\right)=$ $\operatorname{PG}\left(\mathcal{A}_{2}\right)$, and let $\mathcal{B}_{1} \in \mathcal{C}_{\nsim}$ be such that $\mathcal{A}_{1} \leqslant \nsim \mathcal{B}_{1}$. Let $\mathcal{D}_{1}:=\mathcal{D}[\mathcal{B} / \mathcal{A}], \check{\mathcal{D}}:=\check{\mathcal{D}}[\mathcal{B} / \mathcal{A}]$, and let

$$
R_{2}=\bigcup\left\{\left[a_{1}, \ldots, a_{n}\right]_{\mathfrak{g}}:\left(a_{1}, \cdots a_{n}\right) \in R_{\nsim}^{\check{\mathcal{D}}} \backslash R_{\nsim}^{\mathcal{A}_{1}}\right\}
$$

the union of orbits of elements of $R_{\not}^{\check{\mathcal{D}}} \backslash R_{\chi}^{\mathcal{A}_{1}}$ under the action of $\mathfrak{g}$. Let $\mathcal{D}_{2}$ be the structure in $\mathcal{C}_{0}^{\mathfrak{g}}$ with the same universe as $\check{\mathcal{D}}$ and $R_{\mathfrak{g}}^{\mathcal{D}_{2}}=R_{\mathfrak{g}}^{\mathcal{A}_{2}} \cup R_{2}$. Note that for every set $Y$, by construction, $\delta_{\breve{\mathcal{D}}}(Y / Y \cap A)=\delta_{\mathcal{D}_{2}}(Y / Y \cap A)$. In particular, as $\mathcal{A}_{1} \leqslant \nsim \check{\mathcal{D}}$, clearly $\mathcal{A}_{2} \leqslant \mathfrak{g} \mathcal{D}_{2}$. Additionally, by (3) of Lemma 2.13 we get $\operatorname{PG}\left(\mathcal{D}_{2}\right)=\operatorname{PG}(\check{\mathcal{D}})$, and by Lemma 3.12 we know $\operatorname{PG}(\check{\mathcal{D}})=\operatorname{PG}\left(\mathcal{D}_{1}\right)$. Then the structures $\mathcal{D}_{1}, \mathcal{D}_{2}$ are as desired.

Corollary 3.14. $\operatorname{PG}\left(\mathcal{M}_{\mathfrak{g}}\right) \cong \operatorname{PG}\left(\mathcal{M}_{\nsim}\right)$.

Proof. Immediate by Lemma 2.12 .

3.2. Definable reduction. We direct the reader to Definition 2.15 for the precise definition of a definable reduct. In this paper, we will only consider reducts to a single formula, namely, to one of the languages $\mathcal{L}_{\mathfrak{g}}$.

Our goal is to show that whenever $\mathfrak{g}, \mathfrak{h} \leq S_{n}$, then $\mathcal{M}_{\mathfrak{g}}$ is isomorphic to a (proper) definable reduct of $\mathcal{M}_{\mathfrak{h}}$, thus finishing the proof of Theorem 3.9. In case $\mathfrak{g} \leq \mathfrak{h}$, this is easy - we simply "symmetrize" the edges.

Theorem 3.15. Let $\mathfrak{g} \leq \mathfrak{h} \leq S_{n}$. Then the reduct of $\mathcal{M}_{\mathfrak{g}}$ to the formula

$$
\varphi_{R_{\mathfrak{h}}}\left(x_{1}, \ldots, x_{n}\right)=\bigvee_{\sigma \in \mathfrak{h}} R_{\mathfrak{g}}\left(x_{\sigma(1)}, \ldots, x_{\sigma(n)}\right)
$$

is isomorphic to $\mathcal{M}_{\mathfrak{h}}$. Moreover, if $\mathfrak{g} \neq \mathfrak{h}$, the reduct of $\mathcal{M}_{\mathfrak{g}}$ to $\varphi_{R_{\mathfrak{h}}}$ is proper.

Proof. For each $\mathcal{A} \in \overline{\mathcal{C}}_{\mathfrak{g}}$ define $\widehat{\mathcal{A}}$ to be the $\mathcal{L}_{\mathfrak{h}}$-structure which is the reduct of $\mathcal{A}$ to $\varphi_{R_{\mathfrak{h}}}$. We show that P1-P4 of Assumption 2.16 hold with respect to $\mathcal{C}_{\mathfrak{g}}$ and $\mathcal{C}_{\mathfrak{h}}$. By Proposition 2.20 and Corollary 2.22, this will prove the statement.

P1. Let $\mathcal{N} \in \overline{\mathcal{C}}_{\mathfrak{g}}$. Because $\varphi_{R_{\mathfrak{h}}}$ is quantifier free, in fact $\widehat{\mathcal{N}}[A]=\widehat{\mathcal{A}}$ for every substructure $\mathcal{A} \subseteq \mathcal{N}$, regardless of self-sufficiency.

P2. Observe that for any $\mathcal{A} \in \mathcal{C}_{0}^{\mathfrak{g}}$, the inequality $\delta_{\mathfrak{g}}(\mathcal{A}) \leq \delta_{\mathfrak{h}}(\widehat{\mathcal{A}})$ holds. In particular, $\emptyset \leqslant_{\mathfrak{g}} \mathcal{A}$ implies $\emptyset \leqslant_{\mathfrak{h}} \widehat{\mathcal{A}}$. As $\widehat{\mathcal{A}}$ is clearly $\mathfrak{h}$-symmetric, $\widehat{\mathcal{A}} \in \mathcal{C}_{\mathfrak{h}}$.

P3. Let $\mathcal{A} \in \mathcal{C}_{\mathfrak{g}}, \mathcal{B} \in \mathcal{C}_{\mathfrak{h}}$ be such that $\widehat{\mathcal{A}} \leqslant \leqslant_{\mathfrak{h}} \mathcal{B}$. For any $\bar{a}, \bar{b} \in R_{\mathfrak{h}}^{\mathcal{B}} \backslash R_{\mathfrak{h}}^{\widehat{\mathcal{A}}}$, write $\bar{a} \equiv_{\mathfrak{h}} \bar{b}$ if $\bar{b} \in[\bar{a}]_{\mathfrak{h}}$. Let $S$ be a set of representatives for the equivalence classes of $\equiv_{\mathfrak{h}}$. Let $\mathcal{E}$ be the $\mathcal{L}_{\mathfrak{g}}$-structure with the same universe as $\mathcal{B}$ and

$$
R_{\mathfrak{g}}^{\mathcal{E}}=R_{\mathfrak{g}}^{\mathcal{A}} \cup \bigcup\left\{[\bar{a}]_{\mathfrak{g}}: \bar{a} \in S\right\}
$$

Then $\mathcal{A} \leqslant_{\mathfrak{g}} \mathcal{E}$, because $\delta_{\mathcal{E}}(X / A)=\delta_{\mathcal{B}}(X / A)$ for every $X \subseteq B$. As $\widehat{\mathcal{E}}=\mathcal{B}$, in particular $\mathcal{B} \leqslant_{\mathfrak{h}} \widehat{\mathcal{E}}$. 
P4. Assume that there is $\tau \in \mathfrak{h} \backslash \mathfrak{g}$. Let $\mathcal{F} \in \mathcal{C}_{\mathfrak{g}}$ be arbitrary. Define $\mathcal{A}, \mathcal{B}$ to be the structures in $\mathcal{C}_{0}^{\mathfrak{g}}$ with universe $F \cup\left\{a_{1}, \ldots, a_{n}\right\}$ and

$$
\begin{gathered}
R_{\mathfrak{g}}^{\mathcal{A}}=R_{\mathfrak{g}}^{\mathcal{F}} \cup\left[a_{1}, \ldots, a_{n}\right]_{\mathfrak{g}} \\
R_{\mathfrak{g}}^{\mathcal{B}}=R_{\mathfrak{g}}^{\mathcal{F}} \cup\left[a_{1}, \ldots, a_{n}\right]_{\mathfrak{g}} \cup\left[a_{\tau(1)}, \ldots, a_{\tau(n)}\right]_{\mathfrak{g}} .
\end{gathered}
$$

Then $\mathcal{A} \neq \mathcal{B}$, but $\widehat{\mathcal{A}}=\widehat{\mathcal{B}}$.

A more daunting task is reducing the amount of symmetry. The remainder of this section is dedicated to the proof that $\mathcal{M}_{\nsim}$ is isomorphic to a (proper) definable reduct of $\mathcal{M}_{\sim}$. Since the relation " $X$ is isomorphic to a definable reduct of $Y$ " is transitive, combining Theorem 3.15 with a reduction from $\mathcal{M}_{\sim}$ to $\mathcal{M}_{\nsim}$ gives a proof of the second part of Theorem 3.9 via the progression

$$
\mathcal{M}_{\mathfrak{h}} \rightarrow \mathcal{M}_{\sim} \rightarrow \mathcal{M}_{\nsim} \rightarrow \mathcal{M}_{\mathfrak{g}}
$$

In particular, this demonstrates that $\mathcal{M}_{\mathfrak{g}}$ and $\mathcal{M}_{\mathfrak{h}}$ are mutually interpretable (but not necessarily bi-interpretable).

The next thing we do is to isolate desirable properties of a formula with respect to which we will take the reduct. For the remainder of the paper, we will not need to think of $\mathcal{L}_{\mathfrak{g}}$ structures for an arbitrary $\mathfrak{g} \leq S_{n}$, but only of structures in $\mathcal{C}_{0}^{\sim}$ and $\mathcal{C}_{0}^{\chi}$.

To improve readability of the upcoming material, from now on we let $x, y, a, b, r, t, \ldots$ denote tuples of distinct elements. Abusing notation, when appropriate, we identify a tuple $a$ with the set of elements appearing in $a$. In particular, when tuples appear in the context of $\cap, \cup, \backslash \subseteq$, they are thought of as sets. We let $|a|$ denote the length of $a$, or equivalently, as elements appearing in $a$ are distinct, the cardinality of the set of elements appearing in $a$. We write $a b$ for the concatenation of the tuples $a$ and $b$.

Definition 3.16. In the context of a structure $\mathcal{N} \in \mathcal{C}_{\sim}$ and $A, B \subseteq$ fin $N$, say that $B$ is simply algebraic over $A$ if:

- $\delta_{\mathcal{N}}(B / A)=0$

- For every nonempty $X \subset B \backslash A, \delta_{\mathcal{N}}(X / A)>0$.

Definition 3.17. Say that $\mathcal{Q} \in \mathcal{C}_{\sim}$ with universe $a b$-where the elements in $a b$ are pairwise distinct - is sturdy if

(Q1) $|a|=n,|b|>2 n, a \notin R_{\sim}^{\mathcal{Q}}$, and $\delta_{\sim}(\mathcal{Q})=n-1$.

(Q2) $\mathcal{Q}$ is rigid, i.e., the only automorphism of $\mathcal{Q}$ is the identity map.

(Q3) In $\mathcal{Q}$, for every $r \in R_{\sim}^{\mathcal{Q}}$, the set $a b$ is simply algebraic over $r$.

Remark 3.18. Later in the paper, in order to construct sturdy structures, we will induct on $n$, the arity of $R_{\sim}$. To that end, we will use the term $k$-sturdy to indicate that a structure is sturdy, according to Definition 3.17, in the case $n=k$.

An example of a sturdy structure for a ternary $R_{\sim}$ can be found in Lemma 3.34. The following are simple structural consequences to be used later.

Lemma 3.19. Let $\mathcal{Q} \in \mathcal{C}_{\sim}$ be sturdy with universe ab as above. Then

(i) $\mathrm{d}_{\mathcal{Q}}(r)=\delta_{\mathcal{Q}}(r)=n-1$ for every $r \in R_{\sim}^{\mathcal{Q}}$.

(ii) Whenever $X \subseteq a b$ with $\delta_{\mathcal{Q}}(X) \geq n$, the self-sufficient closure of $X$ contains all of $a b$. Hence, if $|X|>n$ or if $|X|=n$ and there is no edge on $X$, then 
$a b \subseteq \Lambda_{\mathcal{Q}}(X)$. In particular, $\Lambda_{\mathcal{Q}}(a)=a b$. (recall that $\Lambda_{\mathcal{Q}}$ is the self-sufficient closure operator)

(iii) For any $B_{1}, B_{2} \subset a b$ with $a b=B_{1} \cup B_{2}$ and $\left|B_{1} \cap B_{2}\right| \leq n$, the structure $\mathcal{Q}$ is not a free join of $B_{1}$ and $B_{2}$ over $A:=B_{1} \cap B_{2}$. That is, $R_{\sim}^{\mathcal{Q}}\left[B_{1}\right] \cup R_{\sim}^{\mathcal{Q}}\left[B_{2}\right] \neq R_{\sim}^{\mathcal{Q}}$.

Proof. The definition of simple algebraicity and (Q3) imply (i) directly. To see (ii), observe that (Q3) implies there is no $X \subseteq a b$ properly containing an edge with $\delta_{\mathcal{Q}}(X)<n$, apart from the entirety of $a b$.

For (iii), assume for a moment $\mathcal{Q}$ is such a free join. Because $|a b|>2 n$, without loss of generality, $\left|B_{1}\right|>n$. Then, by (ii), $0>\delta_{\mathcal{Q}}\left(B_{2} / B_{1}\right)=\delta_{\mathcal{Q}}\left(B_{2} / A\right)$, hence $\delta_{\mathcal{Q}}\left(B_{2}\right)<\delta_{\mathcal{Q}}(A) \leq|A|$. Now, take some $Y$ such that $A \subseteq Y \subseteq B_{1}$ with $|Y|=n$ and note the strict inequality $\delta_{\mathcal{Q}}\left(B_{2} \cup Y\right) \leq \delta_{\mathcal{Q}}\left(B_{2}\right)+|Y \backslash A|<|Y| \leq n$. By (ii), we have $\delta_{\mathcal{Q}}\left(B_{1} / B_{2} \cup Y\right)<0$, implying $\delta_{\sim}(\mathcal{Q})<\delta_{\mathcal{Q}}\left(B_{2} \cup Y\right)<n$. This contradicts $(\mathrm{Q} 1), \delta_{\sim}(\mathcal{Q})=n-1$.

We will prove later that sturdy structures exist. For now, fix a sturdy $\mathcal{Q}$ as in Definition 3.17 above.

Definition 3.20. Define the following

(1) Define $Q(x ; y)$ to be the complete atomic diagram of $a b$ in $\mathcal{Q}$. Explicitly, $Q(a ; b)=\bigwedge_{r \in R \mathcal{Q}} R_{\sim}(r) \wedge \bigwedge_{r \in Q^{n} \backslash R_{\sim}^{\mathcal{Q}}} \neg R_{\sim}(r)$.

(2) Define $Q^{+}(x ; y)$ to be the complete positive atomic diagram of $a b$ in $\mathcal{Q}$. Explicitly, $Q^{+}(a ; b)=\bigwedge_{r \in R_{\sim} \mathcal{O}} R_{\sim}(r)$.

(3) Define $q(x ; y)$ to be the formula stating that $Q(x ; y)$ holds and, whenever $Q^{+}(u ; v)$ holds with $x y \neq u v$, then $|x y \cap u v| \leq n$. This is a universal statement.

(4) For a tuple $c d$ with $|c|=|a|$ and $|d|=|b|$, write $G(c ; d)$ for the set of symmetric edges $\left\{[r]: Q(c ; d) \models R_{\sim}(r)\right\}$.

I.e., $\bigcup G(a ; b)=R_{\sim}^{\mathcal{Q}}$, and $|G(a ; b)|=|a b|-(n-1)$, because $\delta_{\sim}(\mathcal{Q})=n-1$.

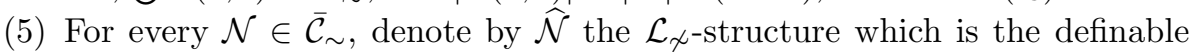
reduct (see Definition 2.15) of $\mathcal{N}$ to the formula

$$
\varphi_{R_{\nsim}}(x):=\exists y q(x ; y) .
$$

Our goal is showing that $\widehat{\mathcal{M}}_{\sim}$ is isomorphic to $\mathcal{M}_{\nsim}$, which we achieve by proving that all properties of Assumption 2.16 hold with respect to $\mathcal{C}_{\sim}$ and $\mathcal{C}_{\not}$, where the map $\mathcal{N} \mapsto \widehat{\mathcal{N}}$ is as defined in Definition 3.20.5 above. Properties P1, P3, P4 are not difficult to prove.

Lemma 3.21. If $\mathcal{A} \leqslant \sim \mathcal{N} \in \overline{\mathcal{C}} \sim$ with $A$ finite, then $\widehat{\mathcal{A}}=\widehat{\mathcal{N}}[A]$. (P1)

Proof. Observe that whenever $\mathcal{N} \models Q^{+}(a ; b)$ with $a \subseteq A$ or $|a b \cap A|>n$, then by Lemma 3.19.ii and $A \leqslant \sim \mathcal{N}$, the self-sufficient closure of $a b \cap A$ is contained in $A$, hence $a b \subseteq A$. So we only need to show that for every $a b \subseteq A$, the structures $\mathcal{A}$ and $\mathcal{N}$ agree on the truth value of $q(a ; b)$.

As a universal statement, $\mathcal{N} \models q(a ; b)$ implies $\mathcal{A} \models q(a ; b)$. If, on the other hand, $\mathcal{N} \models Q(a ; b) \wedge \neg q(a ; b)$, then there exist $c d \neq a b$ such that $\mathcal{N} \models Q^{+}(c ; d)$ and $|a b \cap c d|>n$. In particular, $|c d \cap A|>n$, so by the above paragraph, $c d \subseteq A$ and so also $\mathcal{A} \models \neg q(a ; b)$.

Lemma 3.22. Whenever $\mathcal{A} \in \mathcal{C}_{\sim}, \mathcal{B} \in \mathcal{C}_{\nsim}$ are such that $\widehat{\mathcal{A}} \leqslant \nsim \mathcal{B}$, then there exists some $\mathcal{E} \in \mathcal{C}_{\sim}$ with $\mathcal{A} \leqslant \sim \mathcal{E}$ and $\mathcal{B} \leqslant \nsim \widehat{\mathcal{E}}$. (P3) 
Proof. Denote $S=R_{\varkappa}^{\mathcal{B}} \backslash R_{\chi}^{\widehat{\mathcal{A}}}$, and for each $a \in S$ let $w_{a}$ be a $(|\mathcal{Q}|-n)$-tuple of new elements. Let $G_{a}$ be the set of relations which satisfies $Q\left(a ; w_{a}\right)$. That is, formally, $G_{a}=\bigcup G\left(a ; w_{a}\right)$. Consider the $\mathcal{L}_{\sim}$-structure $\mathcal{E}$ with universe $E=$ $B \cup \bigcup\left\{w_{a}: a \in S\right\}$ and

$$
R_{\sim}^{\mathcal{E}}=R_{\sim}^{\mathcal{A}} \cup \bigcup_{a \in S} G_{a} .
$$

Note that $\mathcal{E}$ is the free join of the $\mathcal{L}_{\sim}$-structures $\left\{\left(B \cup w_{a}, R_{\sim}^{\mathcal{A}} \cup G_{a}\right): a \in S\right\}$ over $\left(B, R_{\sim}^{\mathcal{A}}\right)$, so for any $A \subseteq X \subseteq E$

$$
\begin{aligned}
\delta_{\mathcal{E}}(X / A) & =\delta_{\mathcal{E}}(X \cap B / A)+\delta_{\mathcal{E}}(X / X \cap B) \\
& =\delta_{\mathcal{E}}(X \cap B / A)+\sum_{a \in S} \delta_{\mathcal{E}}\left(X \cap w_{a} / X \cap B\right) \\
& \geq|(X \cap B) \backslash A|-\left|S \cap X^{n}\right|=\delta_{\mathcal{B}}(X \cap B / A) \geq 0 ;
\end{aligned}
$$

hence $\mathcal{A} \leqslant \sim \mathcal{E}$. We have left to show $\mathcal{B} \leqslant \nsim \widehat{\mathcal{E}}$. By Lemma 3.21 we get for free that $\widehat{\mathcal{E}}[A]=\widehat{\mathcal{A}}=\mathcal{B}[A]$. For each $a \in S$, the structure $\mathcal{E}$ can be seen as the free join of $\mathcal{E}\left[E \backslash w_{a}\right]$ and $\mathcal{E}\left[a w_{a}\right]$ over $\mathcal{E}[a]$. Thus, it follows from (iii) of Lemma 3.19 that if $\mathcal{E} \models Q^{+}(c ; d)$, then either $c d \subseteq a w_{a}$ for some $a \in S$, and then by rigidity of $\mathcal{Q}$ it must be that $c=a$ and $d=w_{a}$, or $c d \subseteq \bigcap_{a \in S} E \backslash w_{a}=B$. If the latter holds, then because $R_{\sim}^{\mathcal{E}[B]}=R_{\sim}^{\mathcal{A}}$, in fact $c d \subseteq A$. From this analysis, by construction it follows that $\widehat{\mathcal{E}}[B]=\mathcal{B}$. Additionally, we see that whenever $x$ is such that $\mathcal{E} \models \exists y Q^{+}(x ; y)$, in fact $x \subseteq B$. As all the related tuples in $\widehat{\mathcal{E}}$ are found within $B$, in particular $\widehat{\mathcal{E}}[B] \leqslant \nsim \widehat{\mathcal{E}}$.

Lemma 3.23. For any $\mathcal{F} \in \mathcal{C}_{\sim}$ there exist $\mathcal{A}, \mathcal{B} \in \mathcal{C}_{\sim}$ with $\mathcal{F} \leqslant \sim \mathcal{A}, \mathcal{B}$ and a bijection $f: A \rightarrow B$ fixing $F$ pointwise such that $f$ is an isomorphism between $\widehat{A}$ and $\widehat{B}$, but not an isomorphism between $\mathcal{A}$ and $\mathcal{B}$. $(\mathrm{P} 4)$

Proof. Let $\mathcal{A}, \mathcal{B}$ be the $\mathcal{L}_{\sim}$-structures with universe $F \cup\left\{a_{1}, \ldots, a_{n}\right\}$ and $R_{\sim}^{\mathcal{A}}=R_{\sim}^{\mathcal{F}}$, $R_{\sim}^{\mathcal{B}}=R_{\sim}^{\mathcal{F}} \cup\left[\left(a_{1}, \ldots, a_{n}\right)\right]$, where $a_{1}, \ldots, a_{n}$ are new elements. Take $f$ to be the identity map.

The proof of property $\mathrm{P} 2$ (with $\mathcal{C}_{\sim}$ standing in for $\mathbb{C}_{1}$ and $\mathcal{C}_{\nsim}$ standing in for $\mathbb{C}_{2}$ ), that $\mathcal{A} \in \mathcal{C}_{\sim}$ implies $\widehat{\mathcal{A}} \in \mathcal{C}_{\not}$, is less immediate. Put one way, we are tasked with showing that every structure $\mathcal{A} \in \mathcal{C}_{\sim}$ has only a small number of realizations of $\varphi_{R_{\nsim}}(x)$ (relative to $|A|$ ). Ideally, all the realizations of $q(x, y)$ do not interact with each other, similarly to the construction of $\mathcal{E}$ in the proof of Lemma 3.22. There, we "manufacture" in $\widehat{\mathcal{E}}$ each instance of the relation $a$ appearing in $\mathcal{B}$ by appending a new copy of $Q(a ; b)$ on top of $a$, and so it is easy to see that predimension in the resulting structure $\mathcal{E}$ is bounded from below in terms of predimension in $\mathcal{B}$. However, if there is an edge (i.e., an instance of $R_{\sim}$ ) shared between two (or more) distinct realizations of $q(x, y)$, as the case may be for an arbitrary structure in $\mathcal{C}_{\sim}$, this computation becomes muddled.

Observation 3.24. If $q(a ; b)$ and $q(c ; d)$ hold for $a b \neq c d$ in a structure $\mathcal{N} \in \overline{\mathcal{C}}_{0}$, then $|G(a ; b) \cap G(c ; d)| \leq 1$. This is because, by definition of $q(a ; b)$, as $Q^{+}(c ; d)$ holds, we have $|a b \cap c d| \leq n$ and on $n$ many points there can be at most one symmetric edge. 
Definition 3.25. Let $\mathcal{N} \in \overline{\mathcal{C}}_{0}^{\sim}$. Say that $(a b, c d)$ with $a b \neq c d$ is a weak collision in $\mathcal{N}$ if $\mathcal{N} \models Q^{+}(a ; b) \wedge Q^{+}(c ; d)$ and $G(a ; b) \cap G(c ; d) \neq \emptyset$. Say that $(a b, c d)$ is a collision if in fact $N \models q(a ; b) \wedge q(c ; d)$. For a collision $(a b, c d)$, call the unique element of $G(a ; b) \cap G(c ; d)$ identified in Observation 3.24 the center of the collision.

Define $w_{\mathcal{N}}$ to be the number of weak collisions in $\mathcal{N}$, and define $c_{\mathcal{N}}$ to be the number of collisions in $\mathcal{N}$.

We address the issue by gradually "untangling" an arbitrary structure until all collisions are gone. The order by which we choose to eliminate the collisions will be such that predimension in every intermediate step is still bounded from below in terms of the predimension of the structure we started with. We illustrate this idea and its execution with two instructive examples.

Example 3.26. Let $\mathcal{A} \in \mathcal{C}_{\sim}$ be a structure exemplifying a single collision. Explicitly, $R_{\sim}^{\mathcal{A}}=\bigcup G\left(a_{1} ; b_{1}\right) \cup \bigcup G\left(a_{2} ; b_{2}\right)$ where there is a single $[r] \in G\left(a_{1} ; b_{1}\right) \cap G\left(a_{2} ; b_{2}\right)$, and $a_{1} b_{1} \cap a_{2} b_{2}=r$. By Definition 3.20.5, the reduct $\widehat{\mathcal{A}}$ contains only $a_{1}$ and $a_{2}$ as $R_{\nsim}$-related tuples.

Towards undoing the collision, let us replace $b_{2}$ with a new, external witness to $\varphi_{R_{\nsim}}\left(a_{2}\right)$. Examine the $\mathcal{L}_{\sim}$-structure $\mathcal{B}_{0}$ with universe $A \cup c$, where $c$ is a tuple of new elements, and with $R_{\sim}^{\mathcal{B}_{0}}=R_{\sim}^{\mathcal{A}} \cup \bigcup G\left(a_{2} ; c\right)$. We have $R_{\chi}^{\widehat{\mathcal{A}}} \subseteq R_{\chi}^{\widehat{\mathcal{B}}_{0}}$, so if $\widehat{\mathcal{B}}_{0} \in \mathcal{C}_{\nsim}$, also $\widehat{\mathcal{A}} \in \mathcal{C}_{\nsim}$. However, since we added a complete copy of $\mathcal{Q}$ over $a_{2}$, we have lowered the predimension, i.e. $\delta_{\sim}\left(\mathcal{B}_{0}\right)=\delta_{\sim}(\mathcal{A})-1$, and now it may be that $\mathcal{B}_{0} \notin \mathcal{C}_{\sim}$.

To remedy the problem of $\mathcal{B}_{0}$ having a lower predimension than $\mathcal{A}$, we observe that some symmetric edges in $G\left(a_{2} ; b_{2}\right)$, our "old" configuration witnessing $\varphi_{R_{\nsim}}\left(a_{2}\right)$, are no longer needed. We cannot remove the edge $[r]$ for fear of no longer witnessing $\varphi_{R_{\nsim}}\left(a_{1}\right)$, but if we obtain $\mathcal{B}$ from $\mathcal{B}_{0}$ by removing at least one edge in $G\left(a_{2} ; b_{2}\right) \backslash[r]$, now $\delta_{\sim}(\mathcal{B}) \geq \delta_{\sim}(\mathcal{A})$ and still $R_{\nsim}^{\widehat{\mathcal{A}}} \subseteq R_{\nsim}^{\widehat{\mathcal{B}}}$. Additionally, in $\mathcal{B}$ there are no collisions.

The construction of $\mathcal{B}$ demonstrates a way to "remove" a collision without altering the reduct. This is why we need te upcoming Definition 3.28 , to identify those edges that we can remove from the structure without altering the resulting reduct.

We may repeat this, "outsourcing" the witnessing for $\varphi_{R_{\nsim}}\left(a_{1}\right)$ to a new tuple $d$ and dropping unneeded edges from $G\left(a_{1} ; b_{1}\right)$ (note that unlike before, now $r$ is no longer needed) to obtain a structure $\mathcal{D}$. In $\mathcal{D}$, every tuple witnessing $\varphi_{R_{\nsim}}$ for some $a \in R_{\nsim}^{\widehat{\mathcal{D}}} \supseteq R_{\varkappa}^{\widehat{\mathcal{A}}}$ is completely disjoint from the universe of $\mathcal{A}$ and from every other witnessing tuple, making $\delta_{\widehat{\mathcal{D}}}$ computations easily expressible in terms of $\delta_{\mathcal{D}}$ computations.

The success we achieved in the above example hinges on the ability to offset, in terms of predimension, the addition of a new copy of $\mathcal{Q}$ on top of an existing tuple. For an arbitrary instance of $\varphi_{R_{\nsim}}(x)$, this is not always immediately possible.

Example 3.27. Let $a b$ be a tuple of elements of size $|\mathcal{Q}|$. For every $[r] \in G(a ; b)$, let $c_{r} d_{r}$ be such that $G(a ; b) \cap G\left(c_{r}, d_{r}\right)=\{[r]\}, a b \cap c_{r} d_{r}=r$. Let $\mathcal{A}$ be the structure with universe $a b \cup \bigcup\left\{c_{r} d_{r}:[r] \in G(a ; b)\right\}$ and $R_{\sim}^{\mathcal{A}}=\bigcup\left\{G\left(c_{r} ; d_{r}\right):[r] \in G(a ; b)\right\}$. In words, $\mathcal{A}$ is composed of a "core" $a b$ that is isomorphic to $\mathcal{Q}$, with each edge $r$ in the core being part of a configuration witnessing $\varphi_{R_{\nsim}}\left(c_{r}\right)$ for a tuple $c_{r}$ not contained in the "core". In particular, each edge in the core copy of $\mathcal{Q}$ is the center of a collision. 
If we wish to construct a structure $\mathcal{B}$ where we "outsource" the witness for $\varphi_{R_{\nsim}}(a)$, as we did in the previous example, there is no edge in $G(a ; b)$ we could remove without losing some realization of $\varphi_{R_{\nsim}}(x)$. However, if we first outsource the witness for $\varphi_{R_{\nsim}}\left(c_{r}\right)$, for some $[r] \in G(a ; b)$, then in the resulting structure $\mathcal{B}$ the edge $[r]$ will only be used in the witnessing of $\varphi_{R_{\nsim}}(a)$. At that point, it becomes "safe" to remove $[r]$ from the structure to offset outsourcing the witnessing of $\varphi_{R_{\varkappa}}(a)$. After that is done, we may proceed to outsource the witnessing of the remaining instances of $\varphi_{R_{\nsim}}(x)$, in no particular order.

As the second example demonstrates, the key to employing our strategy is finding a loose end from which to begin the unraveling.

Definition 3.28. For structures $\mathcal{N}, \mathcal{M} \in \overline{\mathcal{C}}_{0}^{\sim}\left(\right.$ or $\mathcal{N}, \mathcal{M} \in \overline{\mathcal{C}}_{0}^{\not}$ ), write $\mathcal{N} \sqsubseteq \mathcal{M}$ to mean that $N \subseteq M$ and $R_{\sim}^{\mathcal{N}} \subseteq R_{\sim}^{\mathcal{M}}$ (or $R_{\nsim}^{\mathcal{N}} \subseteq R_{\chi}^{\mathcal{M}}$ ). In other words, the identity map $\iota: \mathcal{N} \rightarrow \mathcal{M}$ is a homomorphism into $\mathcal{M}$, but not necessarily an embedding.

For $\mathcal{N}, \mathcal{M} \in \overline{\mathcal{C}}_{0}^{\sim}$ with $\mathcal{N} \sqsubseteq \mathcal{M}$, say that a symmetric edge $[r]$ is $\mathcal{N}$-expendable in $\mathcal{M}$, if there exists in $\mathcal{N}$ a unique tuple $a b$ with $\mathcal{M}, \mathcal{N} \models q(a ; b),[r] \in G(a ; b)$, and such that $a b$ takes part in a collision in $\mathcal{M}$. If $\mathcal{N}=\mathcal{M}$ say that $[r]$ is expendable in $\mathcal{M}$.

Note that if $[r]$ is $\mathcal{N}$-expendable in $\mathcal{M}$ and $a b$ is the unique tuple alluded to, then whenever $\mathcal{M} \models q(c ; d)$ with $[r] \in G(c ; d)$ and $a b \neq c d$, then in particular $\mathcal{N} \forall Q^{+}(c ; d)$, or otherwise $c d$ contradicts the uniqueness of $a b$.

The expendable edges are those that can be discarded when resolving collisions. We will show that these must exist in every $\mathcal{A} \in \mathcal{C}_{\sim}$ that has collisions.

While more technically involved in our case, the guiding principle is clearer to explain in terms of an analogy with graphs. In a finite graph in which each vertex has valence at least $k>2$, there are many cycles. Over any one of its vertices, a cycle adds more edges than vertices. I.e, an extension by a cycle has a lower "predimension". If predimension is bounded from below-meaning there is no subset of the graph on which there are more edges than vertices - there cannot be many such extensions, and so there must be a vertex with small valence. This is where we will find an expendable edge.

In this analogy, roughly, vertices are instances of $Q^{+}(x ; y)$ and edges are weak collisions. We define our cycle analogue, and formalize our claim regarding predimension.

Definition 3.29. In the context of some structure $\mathcal{D} \in \overline{\mathcal{C}}_{0}^{\sim}$, let $S$ be a set of symmetric edges in $\mathcal{D}$ and let $L=\left(a_{1} b_{1}, \ldots, a_{k} b_{k}\right)$ be a sequence of distinct (but possibly intersecting) tuples each realizing $q(x ; y)$ in $\mathcal{D}$. Write $G_{i}$ for $G\left(a_{i} ; b_{i}\right)$.

Say that $L$ is an $S$-loop if $S \cap G_{1}, G_{i} \cap G_{i+1}$ are all non-empty, $G_{k} \nsubseteq S$, and

- If $k=1$, then $\left|G_{1} \cap S\right| \geq 2$.

- If $k>1$, letting $[r]$ be the unique (by Observation 3.24) symmetric edge that must be in $G_{k-1} \cap G_{k}$, then $G_{k} \cap\left(S \cup \bigcup_{i=1}^{k-2} G_{i} \backslash\{[r]\}\right)$ is non-empty.

In the next lemma we show that appending an $S$-loop to a structure whose set of edges is $S$ causes a reduction in predimension.

Lemma 3.30. Let $\mathcal{N} \in \overline{\mathcal{C}}_{0}^{\sim}$. Let $\mathcal{D}_{0} \sqsubseteq \mathcal{N}$ be finite, denote $S_{0}=R_{\sim}^{\mathcal{D}_{0}}$, and let $L=\left(a_{1} b_{1}, \ldots, a_{k} b_{k}\right)$ be an $S_{0}$-loop. Denote $G_{i}:=G\left(a_{i} ; b_{i}\right)$ and for each $j \leq k$ denote $D_{j}=D_{0} \cup \bigcup_{i \leq j} a_{i} b_{i}, S_{j}=S_{0} \cup \bigcup_{i \leq j} G_{i}$, and $\mathcal{D}_{j}=\left(D_{j}, S_{j}\right)$. Then,

$$
\delta_{\sim}\left(\mathcal{D}_{k}\right)<\delta_{\sim}\left(\mathcal{D}_{0}\right)
$$


Proof. We think of $\mathcal{D}_{j}$ as the $j$-th stage in the process of appending the loop $L$ to $\mathcal{D}_{0}$, one instance of $q$ at a time.

For each $l<k$, by $\left(a_{l+1} b_{l+1}, G_{l+1}\right) \cong \mathcal{Q}$ and property (Q3) of a sturdy structure (see Definition 3.17), as $G_{l+1}$ intersects $S_{l}$, we have $\left|D_{l+1}\right|-\left|S_{l+1}\right| \leq\left|D_{l}\right|-\left|S_{l}\right|$, and inductively

$$
\left|D_{l}\right|-\left|S_{l}\right| \leq\left|D_{0}\right|-\left|S_{0}\right|
$$

for every $l$. That is almost enough to prove the lemma, but we must have at least one such stage $l$ in which the inequality is strict. We achieve this by slightly altering the order in which we traverse the loop $L$.

Let $0 \leq j<k$ be the least such that $S_{j}$ contains at least two elements of $G_{k}$. We demonstrate that appending $a_{k} b_{k}, G_{k}$ at this stage instead of at the very end brings about the strict inequality we seek, without this changing of order affecting the weak inequalities already established.

We show that $G_{k} \nsubseteq S_{j}$ in order to apply simple algebraicity. If $j=0$, then $G_{k} \not \subseteq S_{j}$ by definition of an $S$-loop of length $k$. Assume $j>0$, i.e., $\left|G_{k} \cap S_{0}\right| \leq 1$. By Observation 3.24 for every $l \leq j$ we have $\left|G_{k} \cap G_{l}\right| \leq 1$, hence $G_{k}$ intersects $S_{l}$ by at most one more symmetric edge than it $\operatorname{did} S_{l-1}$. By choice of $j$, this means $\left|G_{k} \cap S_{j}\right|=2$ precisely. Since $\left|G_{k}\right|>2$, in particular $G_{k} \nsubseteq S_{j}$.

As $S_{j}$ intersects $G_{k}$ in at least two edges, $\left|a_{k} b_{k} \cap D_{j}\right|>n$. Assuming $a_{k} b_{k} \nsubseteq \nsubseteq D_{j}$, by simple algebraicity we have

$$
\left|D_{j} \cup a_{k} b_{k}\right|-\left|S_{j} \cup G_{k}\right|<\left|D_{j}\right|-\left|S_{j}\right| .
$$

If $a_{k} b_{k} \subseteq D_{j}$, the same inequality holds directly by $G_{k} \nsubseteq S_{j}$.

Completing the process, as at the beginning of the proof, for every $l<k$, $\left|D_{l+1} \cup a_{k} b_{k}\right|-\left|S_{l+1} \cup G_{k}\right| \leq\left|D_{l} \cup a_{k} b_{k}\right|-\left|S_{l} \cup G_{k}\right|$, and inductively

$$
\left|D_{k}\right|-\left|S_{k}\right| \leq\left|D_{j} \cup a_{k} b_{k}\right|-\left|S_{j} \cup G_{k}\right| \text {. }
$$

Combining the displayed inequalities, we conclude

$$
\delta_{\sim}\left(\mathcal{D}_{k}\right)=\left|D_{k}\right|-\left|S_{k}\right|<\left|D_{0}\right|-\left|S_{0}\right|=\delta_{\sim}\left(\mathcal{D}_{0}\right)
$$

The next step is to use loops to show that if there are collisions at all, we will be able to find expendable edges (recall Definition 3.28). We achieve this by traversing loops building on non-expendability of edges, until we can loop no more due to predimension constraints.

Lemma 3.31. Let $\mathcal{A} \in \mathcal{C}_{\sim}$ be such that the number of collisions in $\mathcal{A}$ (see Definition 3.25) is positive, i.e., $c_{\mathcal{A}}>0$. Then there exists some symmetric edge $[r]$ which is expendable in $\mathcal{A}$.

Proof. Assume the contrary. Let $\mathcal{A} \in \mathcal{C}_{\sim}$ be such that no symmetric edge in $\mathcal{A}$ is expendable in $\mathcal{A}$. Using the following claim, we will construct $S$-loops. Fix a tuple $a b$ appearing in a collision in $\mathcal{A}$.

Claim 1. Suppose $\mathcal{B} \sqsubseteq \mathcal{A}$ is such that $\mathcal{B} \models Q^{+}(a ; b)$. Denote by $S_{\mathcal{B}}$ the set of symmetric edges in $\mathcal{B}$. If $\left[r_{1}\right] \in G(a ; b)$ is $\mathcal{B}$-expendable in $\mathcal{A}$, then there is an

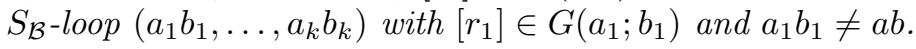

Proof. Recall that no edge in $\mathcal{A}$ is expendable in $\mathcal{A}$. Since in particular $\left[r_{1}\right]$ is not expendable in $\mathcal{A}$, but it is $\mathcal{B}$-expendable in $\mathcal{A}$, there is some $a_{1} b_{1} \neq a b$ with $\mathcal{A} \models q\left(a_{1} ; b_{1}\right)$ such that $\left[r_{1}\right] \in G\left(a_{1} ; b_{1}\right)$. Given $\left[r_{i}\right], a_{i} b_{i}$ such that $\mathcal{A} \models q\left(a_{i} ; b_{i}\right)$ and $\left[r_{i}\right] \in G\left(a_{i} ; b_{i}\right)$, choose arbitrarily some $\left[r_{i+1}\right] \in G\left(a_{i} ; b_{i}\right) \backslash\left\{\left[r_{i}\right]\right\}$. As $a_{i} b_{i}$ appears in 
a collision in $\mathcal{A}$ and, by choice of $\mathcal{A},\left[r_{i+1}\right]$ is not expendable in $\mathcal{A}$, we may choose some $a_{i+1} b_{i+1} \neq a_{i} b_{i}$ such that $\mathcal{A} \models q\left(a_{i+1} ; b_{i+1}\right)$ and $\left[r_{i+1}\right] \in G\left(a_{i+1} b_{i+1}\right)$.

Since $\mathcal{A}$ is finite, there is a large enough $k$ for which $\left(a_{1} b_{1}, \ldots, a_{k} b_{k}\right)$ satisfies the requirements of an $S_{\mathcal{B}}$-loop, maybe apart from $G\left(a_{k} ; b_{k}\right) \nsubseteq S_{\mathcal{B}}$. For such a $k>1$, if $G\left(a_{k} ; b_{k}\right) \subseteq S_{\mathcal{B}}$, then $\left(a_{1} b_{1}, \ldots, a_{k-1} b_{k-1}\right)$ also satisfies the aforementioned requirements, because in this case $\left[r_{k}\right] \in G\left(a_{k-1} ; b_{k-1}\right) \cap S$. Since $\left[r_{1}\right]$ is $\mathcal{B}$-expendable in $\mathcal{A}$, as in the last paragraph of Definition 3.28, we know $\mathcal{B} \not \forall Q^{+}\left(a_{1} ; b_{1}\right)$, hence $G\left(a_{1} ; b_{1}\right) \nsubseteq S_{\mathcal{B}}$. Thus, choosing $k$ minimal such that $\left(a_{1} b_{1}, \ldots, a_{k} b_{k}\right)$ satisfies either bullet of Definition 3.29, we also get that $G\left(a_{k} ; b_{k}\right) \nsubseteq S_{\mathcal{B}}$, which means $\left(a_{1} b_{1}, \ldots, a_{k} b_{k}\right)$ is an $S_{\mathcal{B}}$-loop.

Denote $t=|G(a ; b)|$. Let $X_{0}=a b, S_{0}=G(a ; b)=\left\{\left[r_{1}\right], \ldots,\left[r_{t}\right]\right\}$, and let $\mathcal{B}_{0} \sqsubseteq \mathcal{A}$ be the $\mathcal{L}_{\sim}$-structure on $X_{0}$ with set of symmetric edges $S_{0}$. Note that each of $\left[r_{1}\right], \ldots,\left[r_{t}\right]$ is $\mathcal{B}_{0}$-expendable in $\mathcal{A}$. Recall that by Definition $3.17, \delta_{\sim}\left(\mathcal{B}_{0}\right)=$ $\delta_{\sim}(\mathcal{Q})=n-1$ and, since $|a b|=|\mathcal{Q}|>3 n$, we have $t>2 n+1$.

For $i<n$, given $\mathcal{B}_{i}$ such that at least $2(n-i)$ of $\left[r_{1}\right], \ldots,\left[r_{t}\right]$ are $\mathcal{B}_{i}$-expendable in $\mathcal{A}$, we define inductively an $\mathcal{L}_{\sim}$-structure $\mathcal{B}_{i+1} \sqsubseteq \mathcal{A}$ such that $\mathcal{B}_{i} \sqsubseteq \mathcal{B}_{i+1}$, $\delta_{\sim}\left(\mathcal{B}_{i+1}\right)<\delta_{\sim}\left(\mathcal{B}_{i}\right)$, and at least $2(n-(i+1))$ of $\left[r_{1}\right], \ldots,\left[r_{t}\right]$ remain $\mathcal{B}_{i+1}$-expendable in $\mathcal{A}$.

Denote by $S_{i}$ the set of symmetric edges of $\mathcal{B}_{i}$. Using Claim 1, choose some $S_{i}$-loop $L=\left(a_{1} b_{1}, \ldots, a_{k} b_{k}\right)$ such that $a b \neq a_{1} b_{1}$ and for some $\left[r_{j_{i}}\right]$ that is $\mathcal{B}_{i^{-}}$ expendable in $\mathcal{A},\left[r_{j_{i}}\right] \in G\left(a_{1} ; b_{1}\right)$. Choose $L$ so that $k$ is minimal. Define $\mathcal{B}_{i+1}$ to be the $\mathcal{L}_{\sim}$-structure with universe $X_{i+1}=X_{i} \cup \bigcup_{l=1}^{k} a_{l} b_{l}$ and set of symmetric edges $S_{i+1}=S_{i} \cup \bigcup_{l=1}^{k} G\left(a_{l} ; b_{l}\right)$. By Lemma 3.30, $\delta_{\sim}\left(\mathcal{B}_{i+1}\right)<\delta_{\sim}\left(\mathcal{B}_{i}\right)$.

To proceed with the inductive construction, we only need to show that at least $2(n-i)-2$ of $\left[r_{1}\right], \ldots,\left[r_{t}\right]$ remain $\mathcal{B}_{i+1}$-expendable in $\mathcal{A}$. Maintaining this property assures we can continue the process for $n$ steps.

Claim 2. For some $i<n$, let $\left[r_{j_{i}}\right]$ be the edge to which Claim 1 was applied when constructing $\mathcal{B}_{i+1}$ from $\mathcal{B}_{i}$. Then there is at most one $m \neq j_{i}$ such that $\left[r_{m}\right]$ is $\mathcal{B}_{i}$-expendable in $\mathcal{A}$ but not $\mathcal{B}_{i+1}$-expendable in $\mathcal{A}$.

Proof. Suppose $\left[r_{m}\right]$ is distinct from $\left[r_{j_{i}}\right]$ and that there exists $c d$ a tuple witnessing that $\left[r_{m}\right]$ is $\mathcal{B}_{i}$-expendable in $\mathcal{A}$, but not $\mathcal{B}_{i+1}$-expendable in $\mathcal{A}$. That is, $c d$ is such that $\left[r_{m}\right] \in G(c ; d) \subseteq S_{i+1}$, but $G(c ; d) \nsubseteq S_{i}$. Let $L=\left(a_{1} b_{1}, \ldots, a_{k} b_{k}\right)$ be the $S_{i^{-}}$ loop used to construct $\mathcal{B}_{i+1}$. We claim that $c d=a_{k} b_{k}$. Assume for a contradiction that $c d \neq a_{k} b_{k}$.

By minimality of $k, c d \neq a_{l} b_{l}$ for every $1 \leq l<k$. Moreover, again by minimality of $k, G(c ; d)$ cannot intersect $G\left(a_{l}, b_{l}\right)$ for any $1 \leq l<k-1$. Thus, by Observation 3.24, as $G(c ; d)$ can intersect at most $G\left(a_{k-1} ; b_{k-1}\right)$ and $G\left(a_{k} ; b_{k}\right)$, we have $\left|G(c ; d) \cap\left(S_{i+1} \backslash S_{i}\right)\right| \leq 2$.

Similarly, for any $p<i$, letting $L_{p}=\left(a_{1}^{p} b_{1}^{p}, \ldots, a_{m}^{p} b_{m}^{p}\right)$ be the $S_{p}$-loop used to construct $\mathcal{B}_{p+1}$, we know $c d \neq a_{l}^{p} b_{l}^{p}$ for every $l \leq m$, because $G(c ; d) \nsubseteq S_{p+1}$. As before, by minimality of $m, G(c ; d)$ cannot intersect $G\left(a_{l}^{p} ; b_{l}^{p}\right)$ for any $l<m-1$. So again by Observation 3.24, $\left|G(c ; d) \cap\left(S_{p+1} \backslash S_{p}\right)\right| \leq 2$.

Summing all of these together, we find that $\left|G(c ; d) \cap\left(S_{i+1} \backslash S_{0}\right)\right| \leq 2(i+1)$. By assumption $i<n$ and we know $\left|G(c ; d) \cap S_{0}\right|=1$, so overall $\left|G(c ; d) \cap S_{i+1}\right|<$ $2 n+1<t$, in contradiction to $G(c ; d) \subseteq S_{i+1}$.

Thus, it must be that $c d=a_{k} b_{k}$, so $\left[r_{m}\right] \in G\left(a_{k} ; b_{k}\right)$. In particular, there can be at most $\left|G(a ; b) \cap G\left(a_{k} ; b_{k}\right)\right| \leq 1$ such $m$ as in the statement of the claim. 
Claim 2 guarantees that we can construct up to $\mathcal{B}_{n} \sqsubseteq \mathcal{A}$, but then $\delta_{\mathcal{A}}\left(X_{n}\right) \leq$ $\delta_{\sim}\left(\mathcal{B}_{n}\right) \leq \delta_{\sim}\left(\mathcal{B}_{0}\right)-n<0$, contradicting $\mathcal{A} \in \mathcal{C}_{\sim}$. This proves the lemma.

We can now apply the logic of examples 3.26 and 3.27 to resolve collisions.

Lemma 3.32. Let $\mathcal{A} \in \mathcal{C}_{\sim}$ be such that $c_{\mathcal{A}}>0$. Then there exists some $\mathcal{B} \in \mathcal{C}_{\sim}$ such that

(1) $\widehat{\mathcal{A}} \sqsubseteq \widehat{\mathcal{B}}$

(2) $w_{B}<w_{A}($ recall Definition 3.25)

Proof. As $c_{A}>0$, by Lemma 3.31 we may choose some $[r] \in R_{\sim}^{\mathcal{A}}$ that is expendable in $\mathcal{A}$. Let $a b$ be the unique tuple such that $\mathcal{A} \models q(a, b)$ and $[r] \in G(a ; b)$. Let $w$ be a tuple of new elements with $|w|=|b|$. Define the $\mathcal{L}_{\sim}$-structure $\mathcal{B} \in \mathcal{C}_{0}^{\sim}$ with universe $B=A \cup w$ and

$$
R_{\sim}^{\mathcal{B}}=\left(R_{\sim}^{\mathcal{A}} \backslash[r]\right) \cup \bigcup G(a ; w) .
$$

Note that $\mathcal{B}$ is a free join of $\mathcal{B}[A]$ and $\mathcal{B}[a w]$ over $\mathcal{B}[a]$.

We argue that $\mathcal{B} \in \mathcal{C}_{\sim}$. Let $X \subseteq B$ be such that $\delta_{\mathcal{B}}(X)$ is minimal. If $a \nsubseteq X$, then $\delta_{\mathcal{B}}(X) \geq \delta_{\mathcal{B}}(X \cap A) \geq \delta_{\mathcal{A}}(X \cap A) \geq 0$. If $a \subseteq X$, then $\delta_{\mathcal{B}}(w / X) \leq 0$ and $\delta_{\mathcal{B}}(b / X) \leq 0$, so we may assume $b w \subseteq X$. Now as a free join

$$
\begin{aligned}
\delta_{\mathcal{B}}(X) & =\delta_{\mathcal{B}}(X \cap A)+\delta_{\mathcal{B}}(X \cap a w / a) \\
& =\left(\delta_{\mathcal{A}}(X \cap A)+1\right)-1=\delta_{\mathcal{A}}(X \cap A) \geq 0 .
\end{aligned}
$$

We show that $w_{\mathcal{B}}<w_{\mathcal{A}}$. First, recall that $[r]$ was expendable in $\mathcal{A}$, hence involved in a collision in $\mathcal{A}$. Because $\mathcal{B} \models \neg Q^{+}(a ; b)$, that specific collision no longer exists in $\mathcal{B}$.

Now, observe that if $\mathcal{B} \models Q^{+}(c ; d)$, since $\mathcal{B}[c d]$ is a free join of $\mathcal{B}[c d \cap A]$ and $\mathcal{B}[c d \cap a w]$ over $\mathcal{B}[c d \cap a]$, by (iii) of Lemma 3.19 either $c d \subseteq$ aw or $c d \subseteq A$. If the first occurs, it must be that $G(c ; d)=G(a ; w)$ and by rigidity (Q2 of Definition 3.17) of $\mathcal{Q}, c d=a w$. If the latter occurs, then $\mathcal{B}[A] \models Q^{+}(c ; d)$, hence $\mathcal{A} \models Q^{+}(c ; d)$.

Let $\left(c_{1} d_{1}, c_{2} d_{2}\right)$ be a weak collision in $\mathcal{B}$. If one of the tuples is $a w$, then $G\left(c_{1} ; d_{1}\right) \cap G\left(c_{2} ; d_{2}\right)=\{[a]\}$, which would imply $[a] \in R_{\sim}^{\mathcal{B}}$, hence $[a] \in R_{\sim}^{\mathcal{A}}$. However, since $\mathcal{A} \models q(a ; b)$, in particular $\mathcal{A} \models \neg R_{\sim}(a)$. Then neither tuple is aw, so $\left(c_{1} d_{1}, c_{2} d_{2}\right)$ was already a weak collision in $\mathcal{A}$. We conclude that $w_{B}<w_{A}$.

Lastly, to show $\widehat{\mathcal{A}} \sqsubseteq \widehat{\mathcal{B}}$, we must show that for any $c, \mathcal{A} \models \exists y q(c, y)$ implies $\mathcal{B} \models \exists y q(c, y)$. Noting (iii) of Lemma 3.19 again, if $\mathcal{B} \models Q^{+}(u ; v)$ for some $u v \subseteq B$ then either $u v=a w$ or $\mathcal{A} \models Q^{+}(u ; v)$. Since $a w$ cannot intersect any tuple from $A$ in more than $n$ elements, this is enough so that whenever $\mathcal{A} \models q(c ; d)$ and $\mathcal{B} \models Q^{+}(u ; v)$ with $c d \neq u v$, then $|c d \cap u v| \leq n$. This guarantees that for any $c d \neq a b$ such that $\mathcal{A} \models q(c ; d)$, also $\mathcal{B} \models q(c ; d)$. For the special case $c d=a b$, we no longer have $\mathcal{B} \models Q^{+}(a ; b)$, so $\mathcal{B} \not \models q(a ; b)$. However, we do have $\mathcal{B} \models q(a ; w)$ by construction, and so $\mathcal{B} \models \exists y q(a, y)$ all the same.

Now, showing that we may assume there are no collisions, P2 becomes easy to prove.

Lemma 3.33. If $\mathcal{A} \in \mathcal{C}_{\sim}$, then $\widehat{\mathcal{A}} \in \mathcal{C}_{\nsim}$.

Proof. Assume the statement is false and let $\mathcal{A} \in \mathcal{C}_{\sim}$ contradict it with $w_{\mathcal{A}}$ minimal. We claim that $c_{\mathcal{A}}=0$. Otherwise, by Lemma 3.32 there is some $\mathcal{B} \in \mathcal{C}_{\sim}$ with 
$w_{\mathcal{B}}<w_{\mathcal{A}}$ such that $\widehat{\mathcal{A}} \sqsubseteq \widehat{\mathcal{B}}$. Since $\widehat{\mathcal{A}} \notin \mathcal{C}_{\sim}$, clearly $\widehat{\mathcal{B}} \notin \mathcal{C}_{\nsim}$, contradicting the minimality of $w_{A}$.

Let $Y \subseteq A$ with $\delta_{\widehat{\mathcal{A}}}(Y)<0$ witness $\widehat{\mathcal{A}} \notin \mathcal{C}_{\not}$. Let $W=\{a b: a \subseteq Y, \mathcal{A} \models$ $q(a ; b)\}$ and let $X=Y \cup \cup W$. Since there are no collisions in $\mathcal{A}$, we know that $G(a ; b) \cap G(c ; d)=\emptyset$ whenever $a b, c d \in W$. We compute

$$
\begin{aligned}
\delta_{\mathcal{A}}(X) & \leq|Y|+\left|\bigcup_{a b \in W} b\right|-\left|\bigcup_{a b \in W} G(a ; b)\right| \\
& \leq|Y|+\sum_{a b \in W}(|b|-|G(a ; b)|) \\
& =|Y|-|W| \leq \delta_{\widehat{\mathcal{A}}}(Y)<0
\end{aligned}
$$

This contradicts $\mathcal{A} \in \mathcal{C}_{\sim}$.

Modulo the existence of a sturdy $\mathcal{Q} \in \mathcal{C}_{\sim}$, this finishes the proof of Theorem 3.9. To show the existence of a sturdy $\mathcal{Q}$, we will vary $n$, the arity of $R_{\sim}$, which was fixed up until now. We denote by $\mathcal{L}_{k}$ the language $\mathcal{L}_{\sim}$ for the choice of arity $n=k$. We use $R_{k}$ to denote $R_{\sim}$ in the case $n=k$. In the context of a specific arity, we interpret symbols such as $\leqslant_{k}$ in the obvious way. Write $\mathcal{C}_{\sim}^{k}$ for the collection of $\mathcal{L}_{k}$-structures $\mathcal{A}$ with $\emptyset \leqslant_{k} \mathcal{A}$. As per Remark 3.18 , define an $n$-sturdy structure by modifying Definition 3.17 so that $\mathcal{Q} \in \mathcal{C}_{\sim}^{n}$. Note that (Q1) of the definition depends on $n$ as well.

The proof that an $n$-sturdy structure exists (for $n \geq 3$ ) is by a constructive induction. In the next two lemmas we provide an explicit $n$-sturdy structure for the base case $n=3$, and the induction step.

Lemma 3.34. There exists a 3-sturdy structure $\mathcal{Q}_{3} \in \mathcal{C}_{\sim}^{3}$.

Proof. Consider the structure $\mathcal{Q}_{3}$ with universe $a b=\left\{a_{1}, a_{2}, a_{3}, b_{1}, \ldots, b_{8}\right\}$ and

$$
\begin{aligned}
R_{3}^{\mathcal{Q}_{3}}=\bigcup\{ & {\left[\left(a_{1}, b_{1}, b_{2}\right)\right],\left[\left(a_{2}, b_{2}, b_{3}\right)\right],\left[\left(a_{3}, b_{1}, b_{7}\right)\right], } \\
& {\left[\left(a_{1}, b_{3}, b_{4}\right)\right],\left[\left(a_{2}, b_{4}, b_{5}\right)\right],\left[\left(a_{3}, b_{8}, b_{3}\right)\right], } \\
& {\left[\left(a_{1}, b_{5}, b_{6}\right)\right],\left[\left(a_{2}, b_{6}, b_{7}\right)\right], } \\
& {\left.\left[\left(a_{1}, b_{7}, b_{8}\right)\right]\right\} }
\end{aligned}
$$

It is clear that property (Q1) is satisfied. We prove the other two.

Claim 1. The structure $\mathcal{Q}_{3}$ is rigid. (Q2)

Proof. We will show that each point is $\emptyset$-definable in $\mathcal{Q}_{3}$, and hence fixed by any automorphism. Note that any two edges in $\mathcal{Q}_{3}$ intersect in at most one element.

- $a_{1}$ is the only element appearing in 4 edges.

- $a_{2}$ is the only element appearing in three edges in which $a_{1}$ does not appear.

- $a_{3}$ is the unique element not appearing in an edge with $a_{1}$ or $a_{2}$.

- $\left\{b_{1}, b_{8}\right\}$ is the set of points not sharing an edge with $a_{2}$.

- $b_{7}$ is the unique non- $a_{i}$ element sharing an edge with each element of $\left\{b_{1}, b_{8}\right\}$.

- From $a_{1}, a_{2}, a_{3}, b_{7}$ it is easy to define the rest.

Claim 2. For every $r \in R_{3}^{\mathcal{Q}_{3}}$, the entire structure is simply algebraic over $r$. (Q3) 
Proof. We need to show that if $X \subset a b$ properly contains any edge, then $\delta_{\mathcal{Q}_{3}}(X) \geq 3$. We will show that this is true whenever $|X|>3$, which holds for any set $X$ properly containing an edge.

Let $X \subseteq a b$ be minimal such that $|X|>3$ and $\delta_{\mathcal{Q}_{3}}(X)<3$. We will show that $X$ cannot be a proper subset of $a b$. Note that there must be at least two symmetric edges on $X$. By construction, no two edges intersect in more than one element, so $|X| \geq 5$. Thus, every $x \in X$ must appear in at least two distinct symmetric edges in $X$ or else $X \backslash\{x\}$ contradicts the minimality of $X$.

Therefore, it cannot be that $a_{3} \notin X$, since

$a_{3} \notin X \Longrightarrow b_{1}, b_{8} \notin X \Longrightarrow b_{2}, b_{7} \notin X \Longrightarrow a_{2} \notin X \Longrightarrow b_{4}, b_{5}, b_{6} \notin X$.

and $|X|<3$. Then both edges containing $a_{3}$ must also be in $X$, hence $b_{1}, b_{3}, b_{7}, b_{8} \in$ $X$. Now $b_{1}$ introduces $\left(a_{1}, b_{1}, b_{2}\right)$ into $R_{3}^{\mathcal{Q}_{3}[X]}$, and in turn $b_{2}$ introduces $\left(a_{2}, b_{2}, b_{3}\right)$

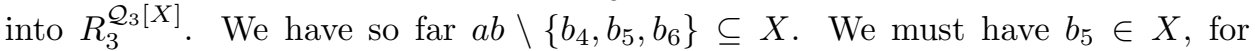
otherwise both $b_{4} \notin X$ and $b_{6} \notin X$, which would imply there is a single edge containing $a_{2}$ in $X$. In turn, $b_{5}$ introduces $\left(a_{2}, b_{4}, b_{5}\right)$ and $\left(a_{1}, b_{5}, b_{6}\right)$ into $R_{3}^{\mathcal{Q}_{3}[X]}$ and we conclude $X=a b$.

The two claims show that $\mathcal{Q}_{3}$ is 3 -sturdy.

Lemma 3.35. If there exists a $k$-sturdy structure $\mathcal{Q}_{k} \in \mathcal{C}_{\sim}^{k}$, then there exists a $k+1$-sturdy structure $\mathcal{Q}_{k+1} \in \mathcal{C}_{\sim}^{k+1}$.

Proof. Let $a b$ be the universe of $\mathcal{Q}_{k}$ where $a=\left(a_{1}, \ldots, a_{k}\right), b=\left(b_{1}, \ldots, b_{l}\right)$. Fix arbitrarily some $r \in R_{k}^{\mathcal{Q}_{k}}$. Since $l>2 k,|b \backslash r| \geq k+1$. Without loss of generality, assume $b_{1}, \ldots, b_{k+1}$ do not appear in $r$.

Let $a_{k+1}, c_{1}, \ldots, c_{k+1}$ be new elements. Define, where if $j>k+1$, then $c_{j}$ stands for $c_{j-(k+1)}$,

$$
\begin{gathered}
\Gamma_{1}=\left\{\left[c_{1} r^{\prime}\right]: r^{\prime} \in R_{k}^{\mathcal{Q}_{k}} \backslash[r]\right\} \\
\Gamma_{2}=\left\{\left[\left(a_{k+1}, b_{i}, c_{i}, \ldots, c_{i+(k-2)}\right)\right]: 1 \leq i \leq k+1\right\}
\end{gathered}
$$

Let $\mathcal{Q}_{k+1}$ be the structure with universe $\left\{a_{1}, \ldots, a_{k+1}, b_{1}, \ldots, b_{l}, c_{1}, \ldots, c_{k+1}\right\}$ and

$$
R_{k+1}^{\mathcal{Q}_{k+1}}=\bigcup \Gamma_{1} \cup\left[c_{2} r\right] \cup \bigcup \Gamma_{2}
$$

Noting $\left|\Gamma_{1}\right|=\left|\left\{\left[r^{\prime}\right]: r^{\prime} \in R_{k}^{\mathcal{Q}_{k}}\right\}\right|-1=l$ and $\left|\Gamma_{2}\right|=k+1$, it is easy to check that (Q1) holds.

Claim 1. $\mathcal{Q}_{k+1}$ is rigid. (Q2)

Proof. Observe

- $c_{1}$ is definable as the only element appearing in at least $l+2$ many symmetric edges in $R_{k+1}^{\mathcal{Q}_{k+1}}$.

- $a_{k+1}$ is definable as the only element appearing in exactly $k+1$ edges of $R_{k+1}^{\mathcal{Q}_{k+1}}$, with exactly two of those not containing $c_{1}$.

- The set $\left\{c_{1}, \ldots, c_{k+1}\right\}$ is definable as the set of points appearing with $a_{k+1}$ in more than one edge.

Then the set $a b$ is definable as the complement of $\left\{a_{k+1}, c_{1}, \ldots, c_{k+1}\right\}$. Also, $R_{k}^{\mathcal{Q}_{k}}$ is a definable relation in $\mathcal{Q}_{k+1}$ as the set of $k$-tuples contained in $a b$ that can be extended to an edge in $R_{k+1}^{\mathcal{Q}_{k+1}}$ by a point from $\left\{c_{1}, \ldots, c_{k+1}\right\}$. Hence, every 
automorphism of $\mathcal{Q}_{k+1}$ fixes $a b$ and $R_{k}^{\mathcal{Q}_{k}}$ set-wise, inducing an automorphism of $\mathcal{Q}_{k}$. By rigidity of $\mathcal{Q}_{k}$, this means every automorphism of $\mathcal{Q}_{k+1}$ fixes $a b$ point-wise.

The edges of $\Gamma_{2}$ are definable as the edges in which $a_{k+1}$ appears. For each $1<i \leq k+1$, the element $c_{i}$ is thus definable over $b_{1}, \ldots, b_{k+1}$, because the elements $b_{i}$ index the edges of $\Gamma_{2}$. So any automorphism of $\mathcal{Q}_{k+1}$ also fixes $\left\{c_{2}, \ldots, c_{k+1}\right\}$ point-wise. That is, the only automorphism of $\mathcal{Q}_{k+1}$ is the identity.

Claim 2. The entire structure is simply algebraic over every $r \in R_{k+1}^{\mathcal{Q}_{k+1}}$.

Proof. As in the proof of Claim 2 in Lemma 3.34, let $X$ be a minimal subset such that $|X|>k+1$ and $\delta_{\mathcal{Q}_{k+1}}(X)<k+1$. In particular, there are at least two symmetric edges on $X$.

We show that $R_{k+1}^{\mathcal{Q}_{k+1}[X]} \nsubseteq \Gamma_{1}$. Otherwise, $c_{1} \in X,|X \cap a b|>k$, and $\delta_{\mathcal{Q}_{k+1}}(X) \geq$ $\delta_{\mathcal{Q}_{k+1}}\left(X \cap c_{1} a b\right)$, so by minimality $X \subseteq c_{1} a b$. If $r \subseteq X$, in particular if $a b \subseteq X$, then

$$
\delta_{\mathcal{Q}_{k+1}}(X)=\left(\delta_{\mathcal{Q}_{k}}(X \cap a b)+1\right)+1 \geq k+1 .
$$

If $r \nsubseteq X$, then $a b \nsubseteq X$, and by sturdiness of $\mathcal{Q}_{k}$ we have $\delta_{\mathcal{Q}_{k}}(X \cap a b) \geq k$ and again

$$
\delta_{\mathcal{Q}_{k+1}}(X)=\delta_{\mathcal{Q}_{k}}(X \cap a b)+1 \geq k+1 .
$$

In any case, this contradicts our choice of $X$.

In case $|X|=k+2$, any two edges on $X$ intersect in $k$ many points. By construction, this is only possible if $R_{k+1}^{\mathcal{Q}_{k+1}[X]} \subseteq \Gamma_{1}$, which we know to be false. Therefore, $|X|>k+2$, and by minimality of $X$, each element in $X$ appears in at least two symmetric edges on $X$. Thus, by construction of $\Gamma_{2}$, the set $\left\{a_{k+1}, c_{2}, \ldots, c_{k+1}\right\}$ is either contained in $X$ or disjoint from $X$. Since $R_{k+1}^{\mathcal{Q}_{k+1}[X]} \nsubseteq \Gamma_{1}$, the set must be contained in $X$. Moreover, $\Gamma_{2} \subseteq R_{k+1}^{\mathcal{Q}_{k+1}[X]}$, so also $c_{1}, b_{1}, \ldots, b_{k+1} \in X$.

Now, unless $a b \subseteq X$, we have $\delta_{\mathcal{Q}_{k+1}}(a b / X)=\delta_{\mathcal{Q}_{k}}(a b / X \cap a b)<0$, implying $k=\delta\left(\mathcal{Q}_{k+1}\right)<\delta_{\mathcal{Q}_{k+1}}(X)$, in contradiction. Conclude that $a b \subseteq X$, i.e., $X$ is the entire universe of $\mathcal{Q}_{k+1}$, which proves the claim.

The two claims show that $\mathcal{Q}_{k+1}$ is $k+1$-sturdy.

Corollary 3.36. For each natural $n \geq 3$ there exists an $n$-sturdy structure $\mathcal{Q}_{n}$.

This is the final component required for the proof of the main theorem.

Theorem 3.9. Whenever $\mathfrak{g}, \mathfrak{h} \leq S_{n}$, then $\operatorname{PG}\left(\mathcal{M}_{\mathfrak{g}}\right) \cong \operatorname{PG}\left(\mathcal{M}_{\mathfrak{h}}\right)$ and $\mathcal{M}_{\mathfrak{h}}$ is isomorphic to a proper definable reduct of $\mathcal{M}_{\mathfrak{g}}$.

Proof. Let $\mathfrak{g}, \mathfrak{h} \leq S_{n}$.

Theorem 3.15 shows that $\mathcal{M}_{\sim}$ is isomorphic to a reduct of $\mathcal{M}_{\mathfrak{g}}$. Taking $\mathcal{Q}$ to be the $n$-sturdy structure guaranteed by Corollary 3.36, lemmas 3.21 to 3.23 and 3.33 show that Assumption 2.16 holds with respect to $\mathcal{C}_{\sim}$ and $\mathcal{C}_{\nsim}$ and reduction to $\varphi_{R_{\nsim}}(x)$, hence $\mathcal{M}_{\nsim}$ is isomorphic to a proper reduct of $\mathcal{M}_{\sim}$. Using Theorem 3.15 again, $\mathcal{M}_{\mathfrak{h}}$ is isomorphic to a reduct of $\mathcal{M}_{\nsim}$. Chaining these reductions, we find that $\mathcal{M}_{\mathfrak{h}}$ is isomorphic to a proper reduct of $\mathcal{M}_{\mathfrak{g}}$.

Finally, by Corollary 3.14, $\operatorname{PG}\left(\mathcal{M}_{\mathfrak{g}}\right) \cong \operatorname{PG}\left(\mathcal{M}_{\nsim}\right) \cong \operatorname{PG}\left(\mathcal{M}_{\mathfrak{h}}\right)$.

An immediate corollary follows.

Corollary 3.37. There is an infinite descending chain of proper reducts with a non-disintegrated pregeometry, beginning with $\mathcal{M}_{\sim}$. 


\section{ACKNOWLEDGMENTS}

The results of this paper were proven as part of the author's PhD candidacy at the Department of Mathematics at Ben-Gurion University of the Negev under the supervision of Dr. Assaf Hasson. The author was partially supported by The Israel Science Foundation grant number 1156/10.

\section{REFERENCES}

[EF11] David M. Evans and Marco S. Ferreira. The geometry of Hrushovski constructions, I: The uncollapsed case. Ann. Pure Appl. Logic, 162(6):474-488, 2011.

[EF12] David M. Evans and Marco S. Ferreira. The geometry of Hrushovski constructions, II. The strongly minimal case. J. Symbolic Logic, 77(1):337-349, 2012.

[HM19] Assaf Hasson and Omer Mermelstein. Reducts of Hrushovski's constructions of a higher geometrical arity. Fund. Math., 247(2):151-164, 2019.

[Hru87] Ehud Hrushovski. Locally modular regular types. In Classification theory (Chicago, IL, 1985), volume 1292 of Lecture Notes in Math., pages 132-164. Springer, Berlin, 1987.

[Hru92] Ehud Hrushovski. Strongly minimal expansions of algebraically closed fields. Israel J. Math., 79(2-3):129-151, 1992.

[Hru93] Ehud Hrushovski. A new strongly minimal set. Ann. Pure Appl. Logic, 62(2):147-166, 1993. Stability in model theory, III (Trento, 1991).

[KS16] Itay Kaplan and Pierre Simon. The affine and projective groups are maximal. Trans. Amer. Math. Soc., 368(7):5229-5245, 2016.

[Mer13] Omer Mermelstein. Geometry preserving reducts of hrushovski's non-collapsed construction. Master's thesis, Ben-Gurion University of the Negev, 2013.

[Rab93] E. D. Rabinovich. Definability of a field in sufficiently rich incidence systems, volume 14 of QMW Maths Notes. Queen Mary and Westfield College School of Mathematical Sciences, London, 1993. With an introduction by Wilfrid Hodges.

(1) Department of Mathematics, Ben-Gurion University of the Negev, Beer-Sheva 8410501, ISRAEL

(2) Department of Mathematics, University of Wisconsin-Madison, Wi 53706, USA

Email address: omer@math.wisc.edu 